\title{
The PSO family: deduction, stochastic analysis and comparison
}

\author{
J.L. Fernández Martínez • E. García Gonzalo
}

Received: 1 June 2008 / Accepted: 2 September 2009 / Published online: 2 October 2009

(C) The Author(s) 2009. This article is published with open access at Springerlink.com

\begin{abstract}
The PSO algorithm can be physically interpreted as a stochastic damped massspring system: the so-called PSO continuous model. Furthermore, PSO corresponds to a particular discretization of the PSO continuous model. In this paper, we introduce a delayed version of the PSO continuous model, where the center of attraction might be delayed with respect to the particle trajectories. Based on this mechanical analogy, we derive a family of PSO versions. For each member of this family, we deduce the first and second order stability regions and the corresponding spectral radius. As expected, the PSO-family algorithms approach the PSO continuous model (damped-mass-spring system) as the size of the time step goes to zero. All the family members are linearly isomorphic when they are derived using the same delay parameter. If the delay parameter is different, the algorithms have corresponding stability regions of any order, but they differ in their respective force terms. All the PSO versions perform fairly well in a very broad area of the parameter space (inertia weight and local and global accelerations) that is close to the border of the second order stability regions and also to the median lines of the first order stability regions where no temporal correlation between trajectories exists. In addition, these regions are fairly similar for different benchmark functions. When the number of parameters of the cost function increases, these regions move towards higher inertia weight values $(w=1)$ and lower total mean accelerations where the temporal covariance between trajectories is positive. Finally, the comparison between different PSO versions results in the conclusion that the centered version (CC-PSO) and PSO have the best convergence rates. Conversely, the centered-progressive version (CPPSO) has the greatest exploratory capabilities. These features have to do with the way these algorithms update the velocities and positions of particles in the swarm. Knowledge of their respective dynamics can be used to propose a family of simple and stable algorithms, including hybrid versions.
\end{abstract}

J.L. Fernández Martínez is a visiting professor at UC-Berkeley (Department of Civil and Environmental Engineering) and Stanford University (Energy Resources Department).

J.L. Fernández Martínez (凶) · E. García Gonzalo

Dept. of Mathematics, University of Oviedo, C/ Calvo Sotelo S/N, Oviedo, Spain

e-mail: jlfm@uniovi.es

E. García Gonzalo

e-mail: espe@uniovi.es 
Keywords Particle swarm optimization $\cdot$ PSO family $\cdot$ Stochastic stability analysis

\section{Introduction}

The particle swarm algorithm applied to optimization problems is very simple: individuals, or particles, are represented by vectors whose length is the number of degrees of freedom of the optimization problem. To start, a population of particles is initialized with random positions $\left(\mathbf{x}_{i}^{0}\right)$ and velocities $\left(\mathbf{v}_{i}^{0}\right)$. The same objective function is used to compute the objective value of each particle. As time advances, the position and velocity of each particle is updated as a function of its objective function value and of the objective function values of its neighbors. At time-step $k+1$, the algorithm updates positions $\left(\mathbf{x}_{i}^{k+1}\right)$ and velocities $\left(\mathbf{v}_{i}^{k+1}\right)$ of the individuals as follows:

$$
\begin{aligned}
\mathbf{v}_{i}^{k+1} & =\omega \mathbf{v}_{i}^{k}+\phi_{1}\left(\mathbf{g}^{k}-\mathbf{x}_{i}^{k}\right)+\phi_{2}\left(\mathbf{l}_{i}^{k}-\mathbf{x}_{i}^{k}\right), \\
\mathbf{x}_{i}^{k+1} & =\mathbf{x}_{i}^{k}+\mathbf{v}_{i}^{k+1},
\end{aligned}
$$

with

$$
\phi_{1}=r_{1} a_{g}, \quad \phi_{2}=r_{2} a_{l}, \quad r_{1}, r_{2} \in U(0,1), \omega, a_{l}, a_{g} \in \mathbb{R},
$$

where $\mathbf{l}_{i}^{k}$ is the $i$ th particle's best position, $\mathbf{g}^{k}$ the global best position on the whole swarm, $\phi_{1}, \phi_{2}$ are random global and local accelerations, and $\omega$ is a real constant called inertia weight. Finally, $r_{1}$ and $r_{2}$ are random numbers uniformly distributed in $(0,1)$, to weight the global and local acceleration constants, $a_{g}$ and $a_{l}$.

In the PSO algorithm, the following vector difference equation is involved for each particle in the swarm:

$$
\left\{\begin{array}{l}
\mathbf{x}_{i}^{k+1}+(\phi-\omega-1) \mathbf{x}_{i}^{k}+\omega \mathbf{x}_{i}^{k-1}=\phi_{1} \mathbf{g}^{k}+\phi_{2} \mathbf{l}_{i}^{k}, \\
\mathbf{x}_{i}^{0}=\mathbf{x}_{i 0}, \\
\mathbf{x}_{i}^{1}=f\left(\mathbf{x}_{i 0}, \mathbf{v}_{i 0}\right),
\end{array}\right.
$$

where $\phi=\phi_{1}+\phi_{2}$ is a random variable with mean $\bar{\phi}=\frac{a_{g}+a_{l}}{2}$ and known statistical distribution (triangular or trapezoidal shape), and $\mathbf{v}_{i 0}, \mathbf{x}_{i 0}$ are the initial velocity and position of particle $i$. Finally, $\mathbf{x}_{i}^{1}$ is the $i$ th particle's position in the first iteration of the PSO algorithm, that can be expressed as a function of $\mathbf{x}_{i 0}$ and $\mathbf{v}_{i 0}$.

Choosing the PSO parameters $\left(\omega, a_{g}, a_{l}\right)$ such that the point $(\omega, \bar{\phi})$ lies inside the first order stability region (Clerc and Kennedy 2002; Trelea 2003; Zheng et al. 2003; Van den Bergh and Engelbrecht 2006, Fernández Martínez et al. 2008)

$$
S_{D}=\{(\omega, \bar{\phi}):|\omega|<1,0<\bar{\phi}<2(\omega+1)\}
$$

causes the particle trajectories to oscillate around their respective center of attraction, namely

$$
\mathbf{o}_{i}^{k}=\frac{a_{g} \mathbf{g}^{k}+a_{l} \mathbf{l}_{i}^{k}}{a_{g}+a_{l}} .
$$

Then, it is easy to understand that the algorithm convergence to the global minimum can be achieved if $\mathbf{o}_{i}^{k}$ is attracting particle $i$ towards the cost function's global minimum. In the case of multimodal functions, entrapment in local minima is possible, and for this reason it 
is very important to have at disposal algorithms with a good balance between exploitation and exploration.

Also, the difference equation (1) can be considered the result of a centered discretization in acceleration

$$
\mathbf{x}_{i}^{\prime \prime}(t) \simeq \frac{\mathbf{x}_{i}(t+\Delta t)-2 \mathbf{x}_{i}(t)+\mathbf{x}_{i}(t-\Delta t)}{\Delta t^{2}},
$$

and a regressive (or backward) scheme in velocity

$$
\mathbf{x}_{i}^{\prime}(t) \simeq \frac{\mathbf{x}_{i}(t)-\mathbf{x}_{i}(t-\Delta t)}{\Delta t}
$$

at time $t=k \in \mathbb{N}$, adopting a unit discretization time step, $\Delta t=1$, applied to the so-called PSO continuous model (Fernández Martínez and García Gonzalo 2008):

$$
\left\{\begin{array}{l}
\mathbf{x}_{i}^{\prime \prime}(t)+(1-\omega) \mathbf{x}_{i}^{\prime}(t)+\phi \mathbf{x}_{i}(t)=\phi_{1} \mathbf{g}(t)+\phi_{2} \mathbf{l}_{i}(t), \quad t \in \mathbb{R} \\
\mathbf{x}_{i}(0)=\mathbf{x}_{i 0} \\
\mathbf{x}_{i}^{\prime}(0)=\mathbf{v}_{i 0} .
\end{array}\right.
$$

This model was derived by Fernández Martínez et al. (2008) using a mechanical analogy: a damped mass-spring system with unit mass, damping factor, $1-w$, and total stiffness constant, $\phi=\phi_{1}+\phi_{2}$. It was also used by Brandstätter and Baumgartner (2002) to optimize electrical engineering problems; nevertheless, this analogy was not completely exploited in their work. Recently, Fernández Martínez et al. (2008) used this analogy to present a systematic study of the PSO mean trajectories. Also, this physical model has given rise to the generalized PSO (GPSO) algorithm (Fernández Martínez and García Gonzalo 2008):

$$
\begin{aligned}
& v(t+\Delta t)=(1-(1-\omega) \Delta t) v(t)+\phi_{1} \Delta t(g(t)-x(t))+\phi_{2} \Delta t(l(t)-x(t)), \\
& x(t+\Delta t)=x(t)+v(t+\Delta t) \Delta t .
\end{aligned}
$$

The GPSO algorithm can also be written in matrix form in terms of the absolute position and velocity of each particle coordinate $(x(t), v(t))$ as follows:

$$
\left(\begin{array}{c}
x(t+\Delta t) \\
v(t+\Delta t)
\end{array}\right)=M_{\mathrm{cr}}\left(\begin{array}{c}
x(t) \\
v(t)
\end{array}\right)+\mathbf{b}_{\mathrm{cr}},
$$

where

$$
M_{\mathrm{cr}}=\left(\begin{array}{cc}
1-\phi \Delta t^{2} & (1-(1-\omega) \Delta t) \Delta t \\
-\phi \Delta t & 1-(1-\omega) \Delta t
\end{array}\right)
$$

and

$$
\mathbf{b}_{\mathrm{cr}}=\left(\begin{array}{c}
\Delta t^{2}\left(\phi_{1} g(t)+\phi_{2} l(t)\right) \\
\Delta t\left(\phi_{1} g(t)+\phi_{2} l(t)\right)
\end{array}\right)
$$

Stochastic stability analysis has recently become a very useful technique to properly understand the particle swarm dynamics and to clarify the PSO convergence properties from a stochastic point of view (Clerc 2006; Kadirkamanathan et al. 2006; Jiang et al. 2007; Poli and Broomhead 2007; Poli 2008; Fernández Martínez and García Gonzalo 2008). Fernández Martínez and García Gonzalo (2009) performed the stochastic analysis of the linear 
continuous and generalized PSO models considering the previous and global best positions of each particle as stochastic processes. The term linear refers to the fact that the interaction between the previous and global best positions and the particle trajectories is done via the covariance functions between the center of attraction, the trajectories and their derivatives (or trajectories in the near past in the GPSO case). This analysis helped to understand the role of the PSO parameters and that of the cost function through the algorithm execution: While PSO parameters control the eigenvalues of the dynamical systems involved, the mean trajectory of the center of attraction and its statistical similarity functions with the trajectories act as force terms to update the mean trajectories and their respective second order moments.

In this paper, we present the deduction and analysis of a family of PSO models. These algorithms come from a delayed version of the continuous PSO model adopting different discretization schemes. For each member of this family, we deduce the first and second order stability regions and the corresponding spectral radii. We show that the most promising parameter zones for each algorithm are located in a broad area close to the upper border of the second order stability region. Also, we show that all the family members are linearly isomorphic when they are deduced using the same delay parameter. If the delay parameter is different, their zones of stability of any order correspond by simple changes of variables, but the algorithms differ in the way they update their respective force terms. Nevertheless, in practice these algorithms perform very differently because of their stochastic character, due to the dependency of the attractors on the visited trajectories, and finally due to the introduction of the delay parameter which is aimed at increasing exploration. Theoretical results are then compared with results from numerical experiments using well known benchmark functions with an increasing degree of numerical difficulties. Also, a numerical comparison between all the PSO family members in terms of their convergence curves and exploration capabilities is performed at corresponding $(w, \bar{\phi})$ points. Even if the no free lunch theorem assures that no algorithm is better than another for any cost function, the numerical simulations showed that PSO and CC-PSO (centered version) are more exploitative versions while the CP-PSO (centered-progressive version) has the greatest exploration capabilities, maintaining a very interesting balance between exploration and exploitation. These features are mainly related to the update of velocities and positions: While CP-PSO acts as a Jacobi system updating velocities and positions at the same time, PSO first updates velocity and then position, and CC-PSO does the opposite using two consecutive centers of attraction. Finally, for most of the benchmark functions, CC-PSO seems to locate the minimum region even faster than PSO.

\section{Methodology for stochastic analysis of trajectories}

Let us consider the trajectories generated by any PSO family member (GPSO, CC-PSO or $\mathrm{CP}-\mathrm{PSO}$ ) as stochastic processes fulfilling the following vector difference equation:

$$
\mathbf{x}(t+\Delta t)-A \mathbf{x}(t)-B \mathbf{x}(t-\Delta t)=C(t) .
$$

Here $A$ and $B$ are random variables, independent from the trajectories $x(t)$ and $x(t-\Delta t)$, and $C(t)$ is a stochastic process that depends on the center of attraction

$$
\mathbf{o}\left(t ; t_{0}\right)=\frac{a_{g} \mathbf{g}\left(t-t_{0}\right)+a_{l} \mathbf{l}\left(t-t_{0}\right)}{a_{g}+a_{l}},
$$


where $\mathbf{g}(t)$ is the global best position, which is common for all the particles of the swarm (or in a neighborhood), and $\mathbf{l}(t)$ is the particle best position, which may be different for each particle in the swarm. In this case, for design purposes we allow the center of attraction $\mathbf{o}\left(t ; t_{0}\right)$ to be delayed a time $t_{0}=k \Delta t$ with respect to the trajectory $\mathbf{x}(t)$. This delay parameter is aimed at increasing exploration and/or at simplifying the final algorithm design. The GPSO algorithm (4) can be easily redesigned introducing the delay parameter $t_{0}$ in $\mathbf{g}(t)$ and $\mathbf{l}(t)$.

Let $x(t)$ be the trajectory of any particle coordinate in the swarm. The first order moment vector

$$
\mu(t+\Delta t)=\left(\begin{array}{c}
E(x(t+\Delta t)) \\
E(x(t))
\end{array}\right)
$$

fulfills the following first order affine dynamical system:

$$
\mu(t+\Delta t)=A_{\mu} \mu(t)+\mathbf{b}_{\mu}(t)
$$

where

$$
A_{\mu}=\left(\begin{array}{cc}
E(A) & E(B) \\
1 & 0
\end{array}\right)
$$

and

$$
\mathbf{b}_{\mu}(t)=\left(\begin{array}{c}
E(C(t)) \\
0
\end{array}\right) .
$$

Stability of the first order moments is related to the eigenvalues of the iteration matrix $A_{\mu}$, and the theoretical asymptotic velocity of convergence ${ }^{1}$ of $x(t)$ towards the mean trajectory of the oscillation center, $E(o(t))$, depends on the spectral radius of first order iteration matrix, $A_{\mu}$ (Fernández Martínez and García Gonzalo 2008, 2009).

Similarly, the non-centered second order moments vector

$$
\mathbf{r}_{2}(t)=\left(\begin{array}{c}
E\left(x^{2}(t)\right) \\
E(x(t) x(t-\Delta t)) \\
E\left(x^{2}(t-\Delta t)\right)
\end{array}\right)
$$

fulfills the following second order affine dynamical system:

$$
\mathbf{r}_{2}(t+\Delta t)=A_{\sigma} \mathbf{r}_{2}(t)+\mathbf{b}_{\mathbf{r}}(t)
$$

where

$$
A_{\sigma}=\left(\begin{array}{ccc}
E\left(A^{2}\right) & 2 E(A B) & E\left(B^{2}\right) \\
E(A) & E(B) & 0 \\
1 & 0 & 0
\end{array}\right)
$$

and

$$
\mathbf{b}_{\mathbf{r}}(t)=\left(\begin{array}{c}
E\left(C^{2}(t)\right)+2 E(A C(t) x(t))+2 E(B C(t) x(t-\Delta t)) \\
E(C(t) x(t)) \\
0
\end{array}\right)
$$

\footnotetext{
${ }^{1}$ The asymptotic velocity of convergence is $-\ln (\rho)$, where $\rho$ is the spectral radius of the iteration matrix, that is, the supremum among the absolute values of the elements in its spectrum (eigenvalues).
} 
The term $E\left(C^{2}(t)\right)$ is related to the oscillation center variability, and the terms $E(A C(t) x(t)), E(B C(t) x(t-\Delta t))$ and $E(C(t) x(t))$ introduce the correlation functions between the oscillation center, $o(t)$, and the trajectories, $x(t)$ and $x(t-\Delta t)$ in the second order affine dynamical system. Similar considerations can be made for the stability and asymptotic velocity of convergence of the second order moments, considering in this case the eigenvalues of iteration matrix $A_{\sigma}$.

Finally, interactions between any two coordinates, $x_{i}(t)$ and $x_{j}(t)$, of any two particles in the swarm can be modeled in the same way. The non-centered second order moments vector

$$
\mathbf{r}_{i j}(t)=\left(\begin{array}{c}
E\left(x_{i}(t) x_{j}(t)\right) \\
E\left(x_{i}(t-\Delta t) x_{j}(t)\right) \\
E\left(x_{i}(t) x_{j}(t-\Delta t)\right) \\
E\left(x_{i}(t-\Delta t) x_{j}(t-\Delta t)\right)
\end{array}\right)
$$

follows a second order affine dynamical system:

$$
\mathbf{r}_{i j}(t+\Delta t)=A_{i j} \mathbf{r}_{i j}(t)+\mathbf{d}_{i j}(t)
$$

where

$$
\begin{aligned}
A_{i j} & =\left(\begin{array}{cccc}
E\left(A^{2}\right) & E(A B) & E(A B) & E\left(B^{2}\right) \\
E(A) & 0 & E(B) & 0 \\
E(A) & E(B) & 0 & 0 \\
1 & 0 & 0 & 0
\end{array}\right), \\
\mathbf{d}_{i j}(t) & =\left(\begin{array}{c}
E\left(x_{i}(t), C_{j}(t)\right) \\
E\left(x_{j}(t), C_{i}(t)\right) \\
0
\end{array}\right),
\end{aligned}
$$

and

$$
\begin{aligned}
d_{1}= & E\left(C_{i}(t) \cdot C_{j}(t)\right)+E(A)\left[E\left(x_{i}(t) \cdot C_{j}(t)\right)+E\left(x_{j}(t) \cdot C_{i}(t)\right)\right] \\
& +E(B)\left[E\left(x_{i}(t-\Delta t) \cdot C_{j}(t)\right)+E\left(x_{j}(t-\Delta t) \cdot C_{i}(t)\right)\right] .
\end{aligned}
$$

The spectral radius of the iteration matrix $A_{i j}$ can be used to analyze the stability of $\mathbf{r}_{i j}(t)$. It is possible to show that (6) and (7) have the same stability conditions.

\section{The GPSO (CR-GPSO) case}

In this section, we present some of the results we have obtained for the GPSO algorithm using this methodology (Fernández Martínez and García Gonzalo 2008, 2009). GPSO corresponds to a regressive (backward) discretization in velocity and a centered discretization in acceleration, so it could be also named CR-GPSO, following the terminology we are going to use in this paper for the other PSO versions.

For the GPSO case with delay $t_{0}$, the variables in difference equation (5) are:

$$
A=2-(1-\omega) \Delta t-\phi \Delta t^{2}
$$




$$
\begin{aligned}
B & =(1-\omega) \Delta t-1, \\
C(t) & =\left(\phi_{1} g\left(t-t_{0}\right)+\phi_{2} l\left(t-t_{0}\right)\right) \Delta t^{2} .
\end{aligned}
$$

The first and second order stability regions are:

$$
\begin{aligned}
& S_{\mathrm{gpso}}^{1}=\left\{(\omega, \bar{\phi}): 1-\frac{2}{\Delta t}<\omega<1,0<\bar{\phi}<\frac{1}{\Delta t^{2}}(2 \omega \Delta t-2 \Delta t+4)\right\}, \\
& S_{\mathrm{gpso}}^{2}=\left\{(\omega, \bar{\phi}): 1-\frac{2}{\Delta t}<\omega<1,0<\bar{\phi}<\phi_{\mathrm{gpso}}(w, \alpha, \Delta t)\right\} .
\end{aligned}
$$

Here $\bar{\phi}=\frac{a_{g}+a_{l}}{2}$ is the total mean acceleration and $\phi_{\mathrm{gpso}}(\omega, \alpha, \Delta t)$ is the analytic expression for the limit hyperbola of second order stability:

$$
\phi_{\mathrm{gpso}}(\omega, \alpha, \Delta t)=\frac{12}{\Delta t} \frac{(1-\omega)(2+(\omega-1) \Delta t)}{4-4(\omega-1) \Delta t+\left(\alpha^{2}-2 \alpha\right)(2+(\omega-1) \Delta t)} .
$$

Also $\alpha=\frac{a_{g}}{\bar{\phi}}=\frac{2 a_{g}}{a_{g}+a_{l}}$ is the ratio between the global acceleration and the total mean acceleration, and varies in the interval [0,2]. Low values of $\alpha$ for the same value of $\bar{\phi}$ imply that the local acceleration is bigger than the global one, and thus the algorithm is more explorative. It is important to note that for $\Delta t=1$ these stability regions do coincide with those shown in previous analyses (Clerc and Kennedy 2002; Trelea 2003; Zheng et al. 2003; Van den Bergh and Engelbrecht 2006; Fernández Martínez et al. 2008; Poli 2008; Fernández Martínez and García Gonzalo 2008).

Figure 1 shows the first and second order stability regions and their corresponding spectral radii for the PSO case. The spectral radii are related to the attenuation of the first and second order trajectories. In the PSO case, the first order spectral radius is zero in $(\omega, \bar{\phi})=(0,1)$. It can be noted that the first order stability zone $\left(S_{\mathrm{gpso}}^{1}\right)$ only depends on $(w, \bar{\phi})$, while the second order stability region $\left(S_{\mathrm{gpso}}^{2}\right)$ depends on $\left(w, a_{g}, a_{l}\right)$. Also, the

(A) First Order Spectral Radius

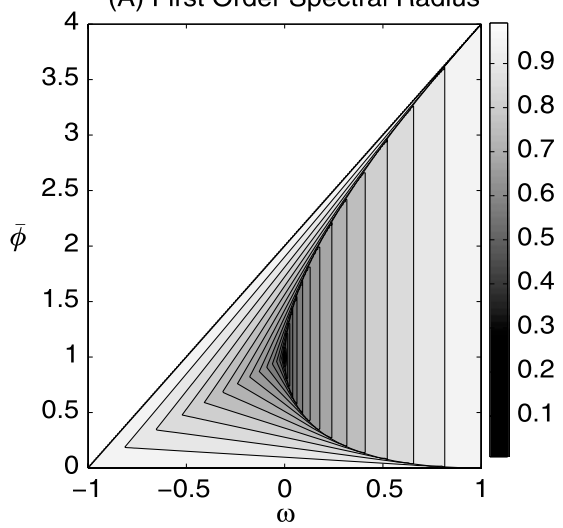

(B) Second Order Spectral Radius

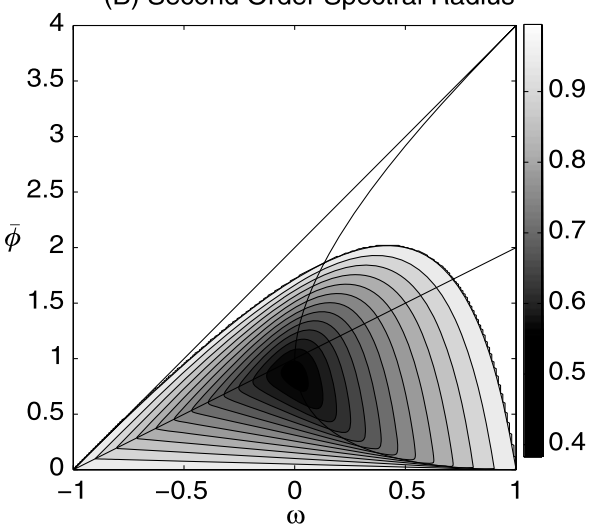

Fig. 1 PSO case. First and second order stability regions for $\Delta t=\alpha=1$, and associated spectral radii. Contour plots can be generalized in the GPSO case, for any couple $(\Delta t, \alpha)$. In the zones where the first spectral radius is close to zero, the attenuation of the mean trajectories is very fast. The same can be said for the second order spectral radius and the variance trajectories 

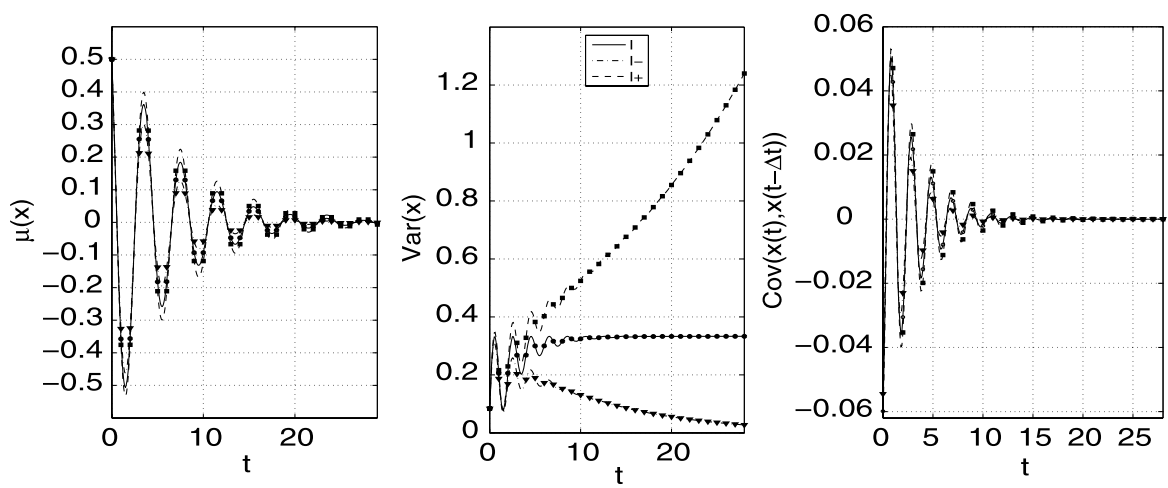

Fig. 2 Mean, variance and covariance trajectories at three points of the line of temporal uncorrelation located below, on and above the upper border of second order stability. The points are: $I=(\omega, \bar{\phi})=\left(\frac{5}{7}, \frac{12}{7}\right)$, $I^{+}=(\omega, \bar{\phi})=(0.75,1.75), I^{-}=(\omega, \bar{\phi})=(0.65,1.65)$

second order stability region is embedded in the first order stability region and depends symmetrically on $\alpha$, reaching its maximum size when $\alpha=1\left(a_{l}=a_{g}\right)$.

Variance trajectories for $(w, \bar{\phi})$ points inside the second order stability go to zero as time advances. On the boundary of the second order stability region, $\bar{\phi}=\phi_{\mathrm{gpso}}(\omega, \alpha, \Delta t)$, variance stabilizes at different constant values depending on the $\left(w, a_{g}, a_{l}\right)$ point. Finally, if $\bar{\phi}>\phi_{\text {gpso }}(\omega, \alpha, \Delta t)$, the variance does not attenuate with time and grows without limit.

Temporal covariance between trajectories, $x(t+\Delta t)$ and $x(t)$, goes very fast to zero on the median line of the first order stability triangle (Fernández Martínez and García Gonzalo 2008, 2009), meaning that trajectories are not temporally correlated. Parameter sets with good performance are close to the intersection of the median line and the hyperbola $\bar{\phi}=$ $\phi_{\text {gpso }}$, that is, trajectories with a high degree of exploration (high variance and temporal uncorrelation). Also, we show that all the PSO versions (including GPSO) perform fairly well in a very broad area in the parameter space (inertia weight and mean acceleration) and these regions are fairly similar for different types of benchmark functions.

To help understand the above mentioned facts which will be relevant in the section devoted to benchmark comparison, Fig. 2 shows the mean, variance and covariance trajectories at three points of the line of temporal uncorrelation located below, on and above the upper border of second order stability. As it can be observed in the three cases, the mean trajectories are stable, the variance trajectories get attenuated if the point is below the border of second order stability, and the temporal covariances between trajectories are symmetric with respect to the time axis in all the cases and go to zero in fewer than ten iterations.

In the next sections, the same methodology will be applied to other PSO versions, which are obtained from the delayed PSO continuous model adopting different discretization schemes in velocity and a centered discretization in acceleration.

\section{CP-GPSO: centered-progressive discretization}

Let us consider a PSO continuous model where the oscillation center may be delayed a time $t_{0}$ with respect to the trajectory $\mathbf{x}_{i}(t)$ :

$$
\mathbf{x}_{i}^{\prime \prime}(t)+(1-\omega) \mathbf{x}_{i}^{\prime}(t)+\phi \mathbf{x}_{i}(t)=\phi_{1} \mathbf{g}\left(t-t_{0}\right)+\phi_{2} \mathbf{l}_{i}\left(t-t_{0}\right), \quad t, t_{0} \in \mathbb{R}
$$


This is a fairly common situation in digital systems.

Let us adopt a centered discretization in acceleration (2) and a progressive scheme in velocity (12) in time $t \in \mathbb{R}$, in order to discretize model (11):

$$
x^{\prime}(t) \simeq \frac{x(t+\Delta t)-x(t)}{\Delta t}
$$

In this case, the acceleration corresponds to a regressive (backward) discretization in velocity

$$
x^{\prime \prime}(t) \simeq \frac{x^{\prime}(t)-x^{\prime}(t-\Delta t)}{\Delta t} .
$$

Considering the PSO continuous model (11) and the above mentioned finite differences schemes, (2) and (12), the following relationship for the velocity is deduced:

$$
\begin{aligned}
v(t)= & \frac{v(t-\Delta t)}{1+(1-\omega) \Delta t}+\frac{\phi_{1} \Delta t}{1+(1-\omega) \Delta t}\left(g\left(t-t_{0}\right)-x(t)\right) \\
& +\frac{\phi_{2} \Delta t}{1+(1-\omega) \Delta t}\left(l\left(t-t_{0}\right)-x(t)\right) .
\end{aligned}
$$

Then

$$
\begin{aligned}
v(t+\Delta t)= & \frac{v(t)}{1+(1-\omega) \Delta t} \\
& +\frac{\phi_{1} \Delta t}{1+(1-\omega) \Delta t}\left(g\left(t+\Delta t-t_{0}\right)-x(t+\Delta t)\right) \\
& +\frac{\phi_{2} \Delta t}{1+(1-\omega) \Delta t}\left(l\left(t+\Delta t-t_{0}\right)-x(t+\Delta t)\right) .
\end{aligned}
$$

Taking into account that

$$
x(t+\Delta t)=x(t)+v(t) \Delta t,
$$

we arrive at the CP-PSO algorithm with delay $t_{0}$ :

$$
\begin{aligned}
v(t+\Delta t)= & \frac{1-\phi \Delta t^{2}}{1+(1-\omega) \Delta t} v(t) \\
& +\frac{\phi_{1} \Delta t}{1+(1-\omega) \Delta t}\left(g\left(t+\Delta t-t_{0}\right)-x(t)\right) \\
& +\frac{\phi_{2} \Delta t}{1+(1-\omega) \Delta t}\left(l\left(t+\Delta t-t_{0}\right)-x(t)\right), \\
x(t+\Delta t)= & x(t)+v(t) \Delta t, \quad t, \Delta t \in \mathbb{R}, \\
x(0)= & x_{0}, \quad v(0)=v_{0} .
\end{aligned}
$$

In this discrete model, the delay $t_{0}$ has to be a multiple of $\Delta t$. Natural choices for $t_{0}$ are $t_{0}=0$ (no delay) or $t_{0}=\Delta t$ (delay 1 ). In this last case, the CP-PSO algorithm simplifies to: 


$$
\begin{aligned}
v(t+\Delta t)= & \frac{1-\phi \Delta t^{2}}{1+(1-\omega) \Delta t} v(t)+\frac{\phi_{1} \Delta t}{1+(1-\omega) \Delta t}(g(t)-x(t)) \\
& +\frac{\phi_{2} \Delta t}{1+(1-\omega) \Delta t}(l(t)-x(t)), \\
x(t+\Delta t)= & x(t)+v(t) \Delta t, \quad t, \Delta t \in \mathbb{R}, \\
x(0)= & x_{0}, \quad v(0)=v_{0} .
\end{aligned}
$$

CP-PSO with delay 1 is a particular case of (14) for a unit time step, $\Delta t=1$. It can be observed that the velocity used to update the position $x(t+\Delta t)$ is $v(t)$ in this case, while in the GPSO case it is $v(t+\Delta t)$ (see (4)). This is one of the major differences between the two algorithms: CP-PSO updates trajectories and velocities at the same time, and taking into account Heisenberg's uncertainty principle, both cannot be known with arbitrary precision. Thus, we can predict in advance that CP-PSO is going to be a very explorative version.

\subsection{The first and second order stability regions}

The following stochastic second order difference equation is obtained for the CP-GPSO algorithm:

$$
x(t+\Delta t)-A_{1} x(t)-B_{1} x(t-\Delta t)=C_{1}(t),
$$

where

$$
\begin{aligned}
A_{1} & =\frac{2+(1-\omega) \Delta t-\phi \Delta t^{2}}{1+(1-\omega) \Delta t}, \\
B_{1} & =\frac{-1}{1+(1-\omega) \Delta t} \in \mathbb{R}, \\
C_{1}(t) & =\frac{\phi_{1} g\left(t-t_{0}\right)+\phi_{2} l_{i}\left(t-t_{0}\right)}{1+(1-\omega) \Delta t} \Delta t^{2} .
\end{aligned}
$$

The first order affine system containing the CP-GPSO mean trajectories is in this case:

$$
\mu(t+\Delta t)=\mathbf{A}_{\mu}^{\mathrm{cp}} \mu(t)+\mathbf{b}_{\mu}^{\mathrm{cp}}(t),
$$

where

$$
\begin{aligned}
\mathbf{A}_{\mu}^{\mathrm{cp}} & =\left(\begin{array}{cc}
E\left(A_{1}\right) & B_{1} \\
1 & 0
\end{array}\right), \\
\mathbf{b}_{\mu}^{\mathrm{cp}}(t) & =\left(\begin{array}{c}
\frac{\bar{\phi} E\left(o\left(t-t_{0}\right)\right)}{1+(1-\omega) \Delta t} \Delta t^{2} \\
0
\end{array}\right),
\end{aligned}
$$

and $A_{1}, B_{1}$ are given respectively by (16) and (17).

The first order stability region of CP-GPSO is the part of the space $(\omega, \bar{\phi})$ where the eigenvalues of matrix $\mathbf{A}_{\mu}^{\mathrm{cp}}$ are in the unit circle. This region is composed of two disjoint zones:

$$
S_{\text {cp-gpso }}^{1}=\left\{(\omega, \bar{\phi}): D_{\mathrm{cp}_{1}}^{1} \cup D_{\mathrm{cp}_{2}}^{1}\right\}
$$




$$
\begin{aligned}
& D_{\mathrm{cp}_{1}}^{1}=\left\{-\infty<\omega<1,0<\bar{\phi}<\frac{2}{\Delta t^{2}}(\Delta t(1-\omega)+2)\right\}, \\
& D_{\mathrm{cp}_{2}}^{1}=\left\{1+\frac{2}{\Delta t}<\omega<+\infty, \frac{2}{\Delta t^{2}}(\Delta t(1-\omega)+2)<\bar{\phi}<0\right\} .
\end{aligned}
$$

The parabola separating the real and complex eigenvalues of $\mathbf{A}_{\mu}^{\mathrm{cp}}$ is given by

$$
\left(\bar{\phi} \Delta t^{2}-(1-\omega) \Delta t-2\right)^{2}=4(1+(1-\omega) \Delta t) .
$$

For $\Delta t=1$, this region of stability becomes

$$
\begin{aligned}
S_{\text {cp-pso }}^{1} & =\left\{(\omega, \bar{\phi}): D_{1} \cup D_{2}\right\}, \\
D_{1} & =\{-\infty<\omega<1,0<\bar{\phi}<2(3-\omega)\}, \\
D_{2} & =\{3<\omega<+\infty, 2(3-\omega)<\bar{\phi}<0\},
\end{aligned}
$$

and the limit parabola is

$$
(\bar{\phi}+\omega-3)^{2}=4(2-\omega) .
$$

The non-centered second order moments fulfill the following second order affine dynamical system:

$$
\mathbf{r}_{2}(t+\Delta t)=A_{\sigma}^{\mathrm{cp}} \mathbf{r}_{2}(t)+\mathbf{b}_{\mathbf{r}}^{\mathrm{cp}}(t)
$$

where

$$
A_{\sigma}^{\mathrm{cp}}=\left(\begin{array}{ccc}
E\left(A_{1}^{2}\right) & 2 B_{1} E\left(A_{1}\right) & B_{1}^{2} \\
E\left(A_{1}\right) & B_{1} & 0 \\
1 & 0 & 0
\end{array}\right)
$$

and

$$
\mathbf{b}_{\mathbf{r}}^{\mathrm{cp}}(t)=\left(\begin{array}{c}
E\left(C_{1}^{2}(t)\right)+2 E\left(A C_{1}(t) x(t)\right)+2 B_{1} E\left(C_{1}(t) x(t-\Delta t)\right) \\
E\left(C_{1}(t) x(t)\right) \\
0
\end{array}\right) .
$$

Terms $E\left(A C_{1}(t) x(t)\right)$ and $E\left(C_{1}(t) x(t-\Delta t)\right)$ introduce the correlation between the trajectories $x(t)$ and $x(t-\Delta t)$ and the attractors $g\left(t-t_{0}\right)$ and $l_{i}\left(t-t_{0}\right)$ into the system.

The eigenvalue analysis of the second order iteration matrix $A_{\sigma}^{\text {cp }}$ allows determining the CP-PSO second order stochastic stability region:

$$
\begin{aligned}
S_{\mathrm{cp}-\mathrm{gpso}}^{2} & =\left\{(\omega, \bar{\phi}): D_{\mathrm{cp}_{1}}^{2} \cup D_{\mathrm{cp}_{2}}^{2}\right\}, \\
D_{\mathrm{cp}_{1}} & =\left\{-\infty<\omega<1,0<\bar{\phi}<\phi_{\mathrm{cp}-\mathrm{pso}}(\omega, \alpha, \Delta t)\right\}, \\
D_{\mathrm{cp}_{2}} & =\left\{1+\frac{2}{\Delta t}<\omega<+\infty, \phi_{\mathrm{cp}-\mathrm{pso}}(\omega, \alpha, \Delta t)<\bar{\phi}<0\right\},
\end{aligned}
$$

which is limited by the hyperbola $\bar{\phi}=\phi_{\text {cp-gpso }}(\omega, \alpha, \Delta t)$ :

$$
\phi_{\text {cp-gpso }}(\omega, \alpha, \Delta t)=\frac{12}{\Delta t} \frac{(1-\omega)((\omega-1) \Delta t-2)}{8(\omega-1) \Delta t+\left(\alpha^{2}-2 \alpha\right)((\omega-1) \Delta t-2)-4} .
$$

As in the GPSO case, $S_{\text {cp-gpso }}^{2}$ is also embedded in the first order stability region, $S_{\text {cp-gpso }}^{1}$, and reaches its maximum size if $\alpha=1\left(a_{g}=a_{l}\right)$. 


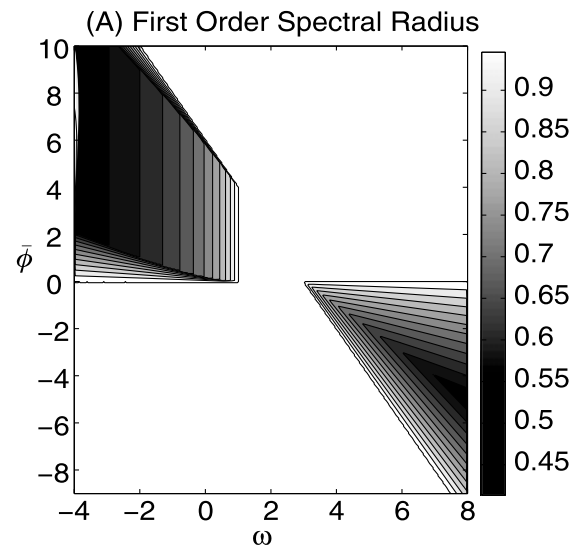

(B) Second Order Spectral Radius

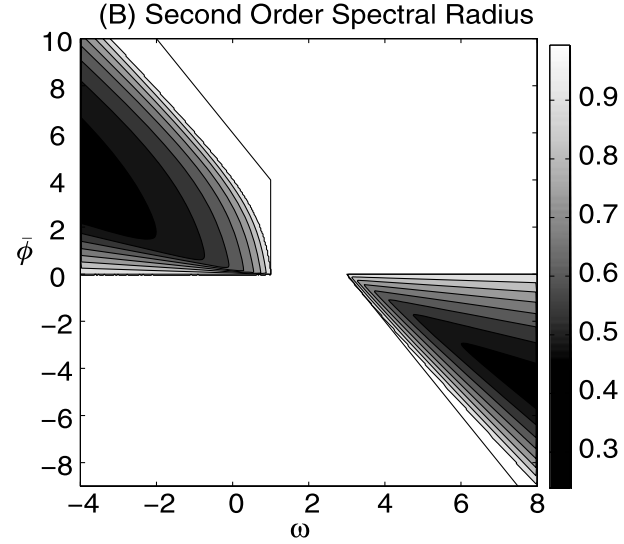

Fig. 3 CP-PSO: First and second order stability regions for $\Delta t=\alpha=1$ and associated spectral radii. Contour plots can be generalized in the GPSO case for any couple $(\Delta t, \alpha)$. Both regions are unbounded and composed of two disjoint zones

Figure 3 shows, for $\Delta t=1$ (CP-PSO case) and $\alpha=1\left(a_{g}=a_{l}\right)$, the first and second order stability regions with the corresponding first and second order spectral radii. Both regions of stability are unbounded and are composed of two disjoint zones. Also, it is important to note that in both cases the first and second order spectral radii are zero at infinity: $(w, \bar{\phi})=(-\infty,+\infty)$ and $(w, \bar{\phi})=(+\infty,-\infty)$. This feature implies a greater exploration capacity for the CP-GPSO algorithm. We intuitively arrived to the same conclusion invoking Heisenberg's principle.

\section{CC-GPSO: centered-centered discretization}

Let us now use a centered discretization in acceleration (2) and a centered scheme in velocity (19) in time $t \in \mathbb{R}$ to approximate the PSO continuous model (11):

$$
x^{\prime}(t) \simeq \frac{x(t+\Delta t)-x(t-\Delta t)}{2 \Delta t} .
$$

The acceleration in this case corresponds to a centered discretization in velocity at times $t+\frac{\Delta t}{2}$ and $t-\frac{\Delta t}{2}$ :

$$
x^{\prime \prime}(t) \simeq \frac{x^{\prime}\left(t+\frac{\Delta t}{2}\right)-x^{\prime}\left(t-\frac{\Delta t}{2}\right)}{\Delta t} .
$$

The following relationship apply:

$$
x^{\prime \prime}(t)=2 \frac{x(t+\Delta t)-x(t)}{\Delta t^{2}}-\frac{v(t)}{\Delta t},
$$

and then

$$
\begin{aligned}
& x(t+\Delta t)=x(t)+\Delta x \Delta t, \\
& \Delta x=\frac{2+(w-1) \Delta t}{2} v(t)+\frac{\Delta t}{2} \phi_{1}\left(l\left(t-t_{0}\right)-x(t)\right)+\frac{\Delta t}{2} \phi_{2}\left(g\left(t-t_{0}\right)-x(t)\right) .
\end{aligned}
$$


The velocity in the CC-GPSO algorithm is updated as follows:

$$
\begin{aligned}
v(t+\Delta t)= & \frac{2+(w-1) \Delta t}{2+(1-w) \Delta t} v(t) \\
& +\frac{\Delta t}{2+(1-w) \Delta t} \sum_{k=0}^{1}\left[\phi_{1}\left(l\left(t-t_{0}+k \Delta t\right)-x(t+k \Delta t)\right)\right. \\
& \left.+\phi_{2}\left(g\left(t-t_{0}+k \Delta t\right)-x(t+k \Delta t)\right)\right] .
\end{aligned}
$$

It can be noted that in CC-PSO the trajectory is updated before the velocity, using two consecutive oscillation centers. This feature is due to the centered scheme adopted in velocity. The CC-GPSO algorithm is deduced adopting $t_{0}=0$ in relationships (20), (21). CC-PSO is obtained from CC-PSO when $\Delta t=1$.

5.1 The first and second order stability regions

The following stochastic second order difference equation is obtained for the CC-GPSO algorithm:

$$
\begin{aligned}
& x(t+\Delta t)-A_{2} x(t)-B_{2} x(t-\Delta t)=C_{2}(t), \\
& A_{2}=2 \frac{2-\phi \Delta t^{2}}{2+(1-\omega) \Delta t}, \\
& B_{2}=\frac{-2+(1-\omega) \Delta t}{2+(1-\omega) \Delta t}, \\
& C_{2}(t)=2 \frac{\phi_{1} g\left(t-t_{0}\right)+\phi_{2} l\left(t-t_{0}\right)}{2+(1-\omega) \Delta t} \Delta t^{2} .
\end{aligned}
$$

It is important to note that the difference equation (22) has a much simpler expression than the CC-PSO algorithm itself.

Applying the same methodology that we have shown above, the first order moments satisfy the dynamical system:

$$
\mu(t+\Delta t)=\mathbf{A}_{\mu}^{\mathrm{cc}} \mu(t)+\mathbf{b}_{\mu}^{\mathrm{cc}}(t),
$$

where

$$
\begin{aligned}
\mathbf{A}_{\mu}^{\mathrm{cc}} & =\left(\begin{array}{cc}
E\left(A_{2}\right) & B_{2} \\
1 & 0
\end{array}\right), \\
\mathbf{b}_{\mu}^{\mathrm{cc}}(t) & =\left(\begin{array}{c}
\frac{2 \bar{\phi} E\left(o\left(t-t_{0}\right)\right)}{2+(1-\omega) \Delta t} \Delta t^{2} \\
0
\end{array}\right) .
\end{aligned}
$$

Here $A_{2}$ and $B_{2}$ are given respectively by (23) and (24).

The first order stability region of the CC-GPSO is:

$$
S_{\mathrm{cc}-\mathrm{gpso}}^{1}=\left\{(\omega, \bar{\phi}): \omega<1,0<\bar{\phi}<\frac{4}{\Delta t^{2}}\right\} .
$$


The curve separating real and complex eigenvalues of $\mathbf{A}_{\mu}^{c c}$ in the first stability region is the ellipse:

$$
\left(\frac{\omega-1}{\frac{2}{\Delta t}}\right)^{2}+\left(\frac{\bar{\phi}-\frac{2}{\Delta t^{2}}}{\frac{2}{\Delta t^{2}}}\right)^{2}=1 .
$$

The spectral radius is zero at the point $(\omega, \bar{\phi})=\left(1-\frac{2}{\Delta t}, \frac{2}{\Delta t^{2}}\right)$.

The non-centered second order moments satisfy the following second order affine dynamical system:

$$
\mathbf{r}_{2}(t+\Delta t)=A_{\sigma}^{\mathrm{cc}} \mathbf{r}_{2}(t)+\mathbf{b}_{\mathbf{r}}^{\mathrm{cc}}(t)
$$

where

$$
A_{\sigma}^{\mathrm{cc}}=\left(\begin{array}{ccc}
E\left(A_{2}^{2}\right) & 2 B_{2} E\left(A_{2}\right) & B_{2}^{2} \\
E\left(A_{2}\right) & B_{2} & 0 \\
1 & 0 & 0
\end{array}\right)
$$

and

$$
\mathbf{b}_{\mathbf{r}}^{\mathrm{cc}}(t)=\left(\begin{array}{c}
E\left(C_{2}^{2}(t)\right)+2 E\left(A_{2} C_{2}(t) x(t)\right)+2 B_{2} E\left(C_{2}(t) x(t-\Delta t)\right) \\
E\left(C_{2}(t) x(t)\right) \\
0
\end{array}\right)
$$

where $A_{2}, B_{2}$ and $C_{2}(t)$ are given respectively by (23), (24) and (25).

The same considerations as mentioned above apply for the terms $E\left(C_{2}^{2}(t)\right)$, $E\left(A_{2} C_{2}(t) x(t)\right)$ and $E\left(C_{2}(t) x(t-\Delta t)\right)$.

The second order stability region is:

$$
S_{\text {cc-gpso }}^{2}=\left\{(\omega, \bar{\phi}): \omega<1,0<\bar{\phi}<\phi_{\text {cc-gpso }}(\omega, \alpha, \Delta t)\right\}
$$

where $\bar{\phi}=\phi_{\text {cc-gpso }}(\omega, \alpha, \Delta t)$ is the limit hyperbola of second order stability:

$$
\phi_{\text {cc-gpso }}(\omega, \alpha, \Delta t)=\frac{12}{\Delta t} \frac{(\omega-1)}{3(\omega-1) \Delta t+2 \alpha-\alpha^{2}-2} .
$$

As in the other two cases, the region $S_{\text {cc-gpso }}^{2}$ is embedded in $S_{\text {cc-gpso }}^{1}$ and reaches its maximum size for $\alpha=1$. CC-PSO is the particular case where the time step is $\Delta t=1$. Figure 4 shows the first and second order stability regions of the CC-PSO case $(\Delta t=1)$ with the associated spectral radii. For the second order region the parameter $\alpha$ has been set to 1 in this case. As it can be observed, both regions of stability are unbounded along the inertia-weight axis.

\section{The $\beta$-GPSO and the corresponding regions of stability}

In order to link together all the PSO versions presented above, let us consider a centered scheme in acceleration, and introduce a real parameter $\beta \in[0,1]$ in the velocity discretization as follows:

$$
x^{\prime}(t) \simeq \frac{(\beta-1) x(t-\Delta t)+(1-2 \beta) x(t)+\beta x(t+\Delta t)}{\Delta t} .
$$



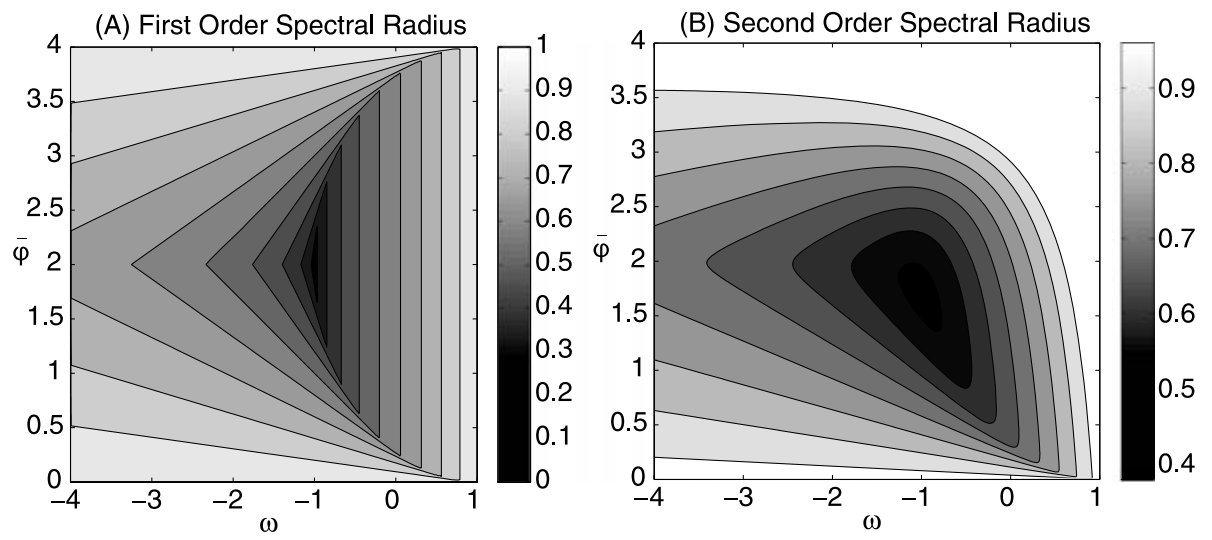

Fig. 4 CC-PSO. First and second order stability regions for CC-PSO and associated spectral radii. Both regions are unbounded along the $w$ axis

Then, for $\beta=1$ the discretization is progressive (CP-GPSO), for $\beta=0.5$ it is centered (CCGPSO), and for $\beta=0$ it is regressive (CR-GPSO or just GPSO). Considering a centered scheme in acceleration, we deduce the $\beta$-GPSO algorithm, that can be written in terms of the absolute position and velocity $(x(t), v(t))$ as follows:

$$
\left(\begin{array}{c}
x(t+\Delta t) \\
v(t+\Delta t)
\end{array}\right)=M_{\beta}\left(\begin{array}{c}
x(t) \\
v(t)
\end{array}\right)+b_{\beta},
$$

where

$$
M_{\beta}=\left(\begin{array}{cc}
1+(\beta-1) \Delta t^{2} \phi & \Delta t(1+(\beta-1)(1-w) \Delta t) \\
\Delta t \phi \frac{(1-\beta) \beta \Delta t^{2} \phi-1}{1+(1-w) \beta \Delta t} & \left(1-\beta \Delta t^{2} \phi\right) \frac{1+(1-w)(\beta-1) \Delta t}{1+(1-w) \beta \Delta t}
\end{array}\right),
$$

and

$$
b_{\beta}=\left(\begin{array}{c}
\Delta t^{2}(1-\beta)\left(\phi_{1} g\left(t-t_{0}\right)+\phi_{2} l\left(t-t_{0}\right)\right) \\
\Delta t \frac{\phi_{1}(1-\beta)\left(1-\beta \Delta t^{2} \phi\right) g\left(t-t_{0}\right)+\beta \phi_{1} g\left(t+\Delta t-t_{0}\right)+\phi_{2}(1-\beta)\left(1-\beta \Delta t^{2} \phi\right) l\left(t-t_{0}\right)+\phi_{2} \beta l\left(t+\Delta t-t_{0}\right)}{1+(1-w) \beta \Delta t}
\end{array}\right) .
$$

Then, the CC-PSO and CP-PSO algorithms can be written in matrix form as follows:

- CC-PSO $(\beta=1 / 2)$ :

$$
\begin{aligned}
M_{\mathrm{CC}} & =\left(\begin{array}{cc}
1-\frac{\Delta t^{2} \phi}{2} & \frac{\Delta t}{2}(2-(1-w) \Delta t) \\
\Delta t \phi \frac{\left(\Delta t^{2} \phi-4\right)}{4+2(1-w) \Delta t} & \frac{(2-(1-w) \Delta t)\left(2-\Delta t^{2} \phi\right)}{4+2(1-w) \Delta t}
\end{array}\right), \\
b_{\mathrm{CC}} & =\left(\begin{array}{c}
\frac{\Delta t^{2}}{2}\left(\phi_{1} g\left(t-t_{0}\right)+\phi_{2} l\left(t-t_{0}\right)\right) \\
\Delta t \frac{\left(2-\Delta t^{2} \phi\right) \phi_{1} g\left(t-t_{0}\right)+2 \phi_{1} g\left(t+\Delta t-t_{0}\right)+\phi_{2}\left(\left(2-\Delta t^{2} \phi\right) l\left(t-t_{0}\right)+2 l\left(t+\Delta t-t_{0}\right)\right)}{4+2(1-w) \Delta t}
\end{array}\right) .
\end{aligned}
$$

- $\operatorname{CP}-\mathrm{PSO}(\beta=1)$ :

$$
M_{\mathrm{CP}}=\left(\begin{array}{cc}
1 & \Delta t \\
\frac{-\Delta t \phi}{1+(1-w) \Delta t} & \frac{1-\Delta t^{2} \phi}{1+(1-w) \Delta t}
\end{array}\right)
$$




$$
b_{\mathrm{CP}}=\left(\begin{array}{c}
0 \\
\frac{\Delta t\left(\phi_{1} g\left(t+\Delta t-t_{0}\right)+\phi_{2} l\left(t+\Delta t-t_{0}\right)\right)}{1+(1-w) \Delta t}
\end{array}\right) .
$$

It can be observed that all the versions with $\beta \in(0,1)$ update the velocity using two consecutive centers of attraction. This situation has been already encountered in the CCPSO case where $\beta=1 / 2$.

Considering the PSO continuous model (3) and the above mentioned finite difference schemes, (2) and (26), the following stochastic second order difference equation is obtained:

$$
x(t+\Delta t)-A_{\beta} x(t)-B_{\beta} x(t-\Delta t)=C_{\beta}(t),
$$

where

$$
\begin{aligned}
A_{\beta} & =\frac{2-\phi \Delta t^{2}-(1-\omega)(1-2 \beta) \Delta t}{1+(1-\omega) \beta \Delta t,}, \\
B_{\beta} & =-\frac{1+(1-\omega)(\beta-1) \Delta t}{1+(1-\omega) \beta \Delta t,}, \\
C_{\beta}(t) & =\frac{\phi_{1} g\left(t-t_{0}\right)+\phi_{2} l\left(t-t_{0}\right)}{1+(1-\omega) \beta \Delta t} \Delta t^{2} .
\end{aligned}
$$

Applying the same methodology, we deduced the first order stability region of the $\beta$-PSO that depends on $\beta$ :

1. If $0 \leq \beta \leq 0.5$,

$$
S_{\beta \text {-pso }}^{1}=\left\{(\omega, \bar{\phi}): 1-\frac{2}{(1-2 \beta) \Delta t}<\omega<1,0<\bar{\phi}<\frac{2(2+(1-\omega)(2 \beta-1) \Delta t)}{\Delta t^{2}}\right\} .
$$

The straight line, $\bar{\phi}=\frac{2(2+(1-\omega)(2 \beta-1) \Delta t)}{\Delta t^{2}}$, always passes through the point $(\omega, \bar{\phi})=$ $\left(1, \frac{4}{\Delta t^{2}}\right)$ for any $\beta$.

2. If $0.5<\beta \leq 1$, this region is composed of two different disjoint zones, and is:

$$
\begin{aligned}
S_{\beta \text {-pso }}^{1} & =\left\{(\omega, \bar{\phi}): D_{\beta_{1}}^{1} \cup D_{\beta_{2}}^{1}\right\}, \\
D_{\beta_{1}}^{1} & =\left\{(\omega, \bar{\phi}):-\infty<\omega<1,0<\bar{\phi}<\frac{2(2+(1-\omega)(2 \beta-1) \Delta t)}{\Delta t^{2}}\right\}, \\
D_{\beta_{2}}^{1} & =\left\{1+\frac{2}{(2 \beta-1) \Delta t}<\omega<+\infty, \frac{2(2+(1-\omega)(2 \beta-1) \Delta t)}{\Delta t^{2}}<\bar{\phi}<0\right\} .
\end{aligned}
$$

3. The border line separating the real and complex roots in the first stability regions is an ellipse for $\beta \in(0,1)$, and only at the limits $\beta=0$ (GPSO) and $\beta=1$ (CP-GPSO) this line becomes a parabola. $\beta$-PSO is a particular case when the time step is $\Delta t=1$.

The second order stability region shows a similar behavior as the first order stability region, it is embedded in the first order region and has as the limit of second order stability the line:

$$
\begin{aligned}
& \phi_{\beta \text {-pso }}(\beta, \omega, \alpha, \Delta t) \\
& \quad=\frac{12}{\Delta t} \frac{(1-\omega)((\omega-1)(2 \beta-1) \Delta t-2)}{(4(-1+(\omega-1)(1+\beta) \Delta t)+(2-\alpha) \alpha(2+(1-\omega)(2 \beta-1) \Delta t)} .
\end{aligned}
$$


(A) First Order Spectral Radius

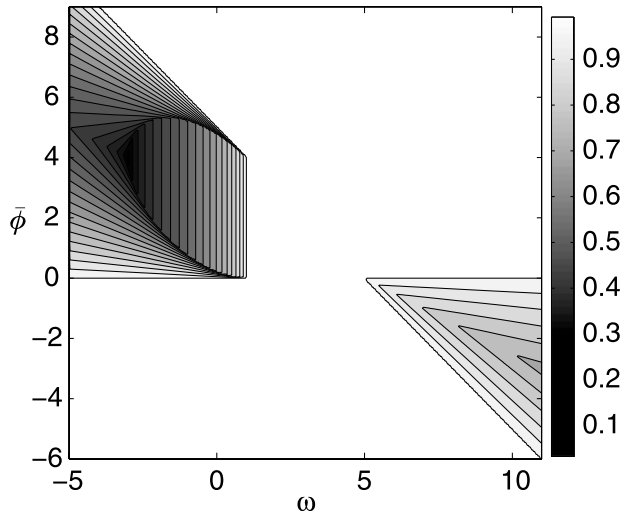

(B) Second Order Spectral Radius

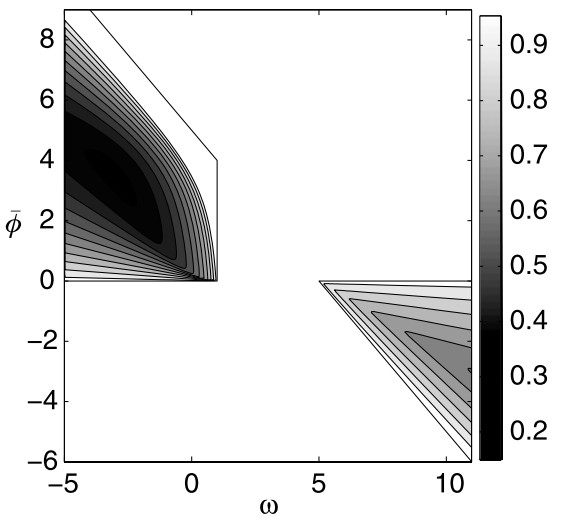

Fig. 5 First and second order stability regions for $\beta$-PSO $(\beta=0.75)$ and associated spectral radius

For instance, Fig. 5 shows the first and second order stability regions with the associated spectral radii for $\beta=0.75$. The similarity with the CP-PSO case $(\beta=1)$ can be observed. In fact, when $0 \leq \beta \leq 0.5$, the regions of first and second order stability are single domains, evolving from the GPSO towards the CC-PSO type, and when $0.5<\beta \leq 1$ both regions are composed of two zones, evolving towards the CP-PSO stability regions as $\beta$ increases.

\section{Correspondence rules}

Since all the PSO algorithms presented in this paper come from different finite difference schemes of the same continuous model (11), there should be a correspondence between discrete trajectories for the different PSO versions. The change of variables to make a $\beta$ PSO version with parameters $a_{g}, a_{l}, w, \Delta t$ correspond to a standard PSO $(\Delta t=1)$ with parameters $b_{g}, b_{l}$, and $\gamma$ is as follows:

$b_{g}=\frac{\Delta t^{2}}{1+(1-w) \beta \Delta t} a_{g}, \quad b_{l}=\frac{\Delta t^{2}}{1+(1-w) \beta \Delta t} a_{l}, \quad \gamma=\frac{1+(1-w)(\beta-1) \Delta t}{1+(1-w) \beta \Delta t}$.

These relationships can be deduced identifying the coefficients (28) and (29) in difference equation (27) with the corresponding coefficients of the standard PSO, (8) and (9), for $\Delta t=1$. The correspondence rules (31) also make the second terms of the corresponding difference equations equal if the delay parameter $t_{0}$ is the same for all the versions. In this case, the equivalence is absolute and the different versions are linearly isomorphic. If the delay parameter $t_{0}$ is not the same, the different versions have corresponding stability regions of any order but they have non-corresponding force terms.

Figure 6 shows the correspondence (31) between the first order trajectories of CC-PSO and CP-PSO with those of the PSO. The following can be observed:

1. In the CC-PSO and CP-PSO cases, the regions of first order stability become unbounded, while in the PSO case this zone is limited by the straight line $\bar{\phi}=2(\omega+1)$, which is, in fact, due to the unit time step adopted (when $\Delta t$ goes to zero, this line goes to infinity).

2. For the different PSO versions, there is a correspondence between the different zones of first order trajectories: complex zone, real symmetrical zigzagging zone, real asymmetric 
(A) CC-PSO

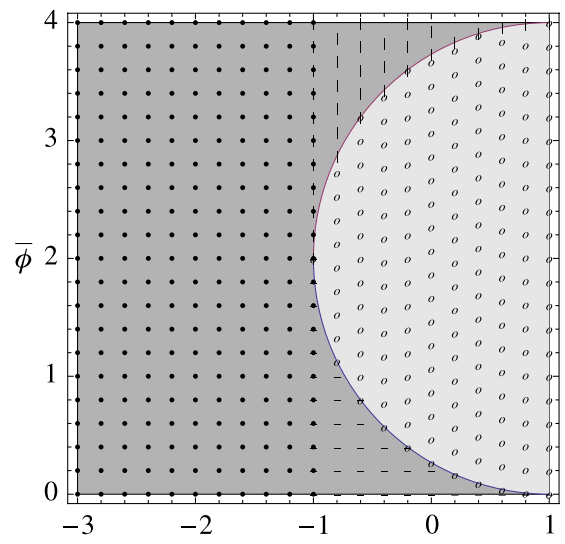

(C) CP-PSO

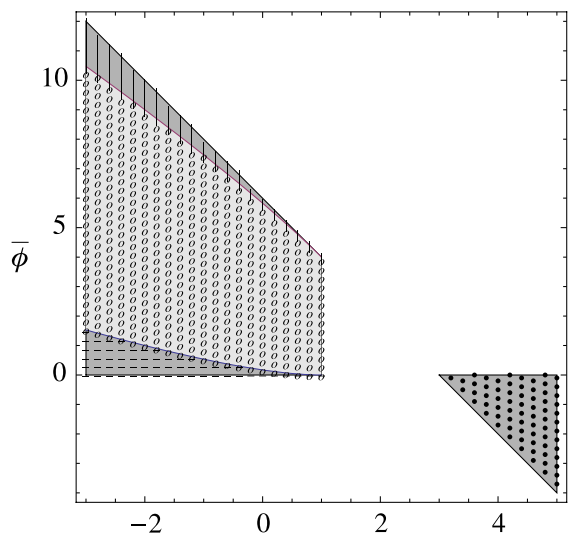

(B) PSO

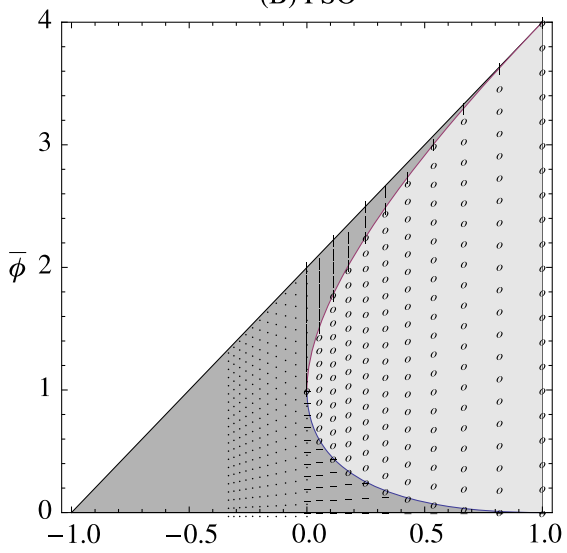

(D) PSO

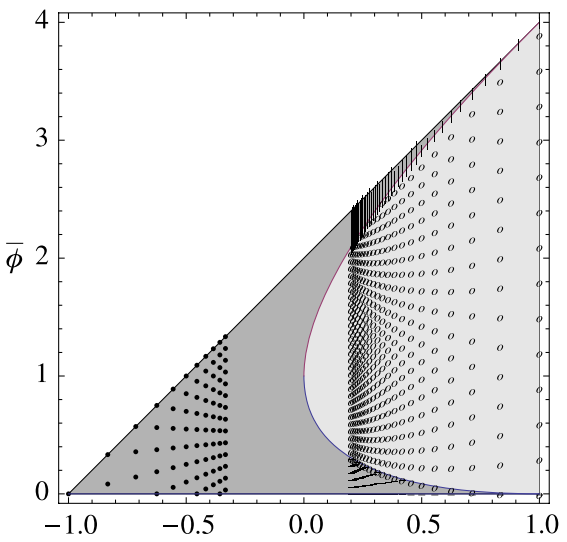

Fig. 6 Corresponding trajectories between PSO and CC-PSO ((A) and (B)) and PSO and CP-PSO $((\mathbf{C})$ and $(\mathbf{D}))$. Observe that in the CP-PSO case points corresponding to PSO points which are in the neighborhood of $(\omega, \bar{\phi})=(0,1)$ are close to $(\omega, \bar{\phi}) \rightarrow(-\infty,+\infty) \cup(\omega, \bar{\phi}) \rightarrow(+\infty,-\infty)$. This means that CP-PSO has greater exploratory capacity than PSO and CC-PSO

oscillating zone and real non-oscillating zone (Fernández Martínez et al. 2008). In the CC-PSO case, the real asymmetric zigzagging zone becomes unbounded (Figs. 6(A) and 6(B)). In the CP-PSO case, the four zones become unbounded (Figs. 6(C) and 6(D)).

3. The correspondence rules between PSO and CP-PSO are:

$$
\bar{\phi}_{\mathrm{cp}}=\frac{\bar{\phi}_{\mathrm{pso}}}{w_{\mathrm{pso}}}, \quad w_{\mathrm{cp}}=\frac{2 w_{\mathrm{pso}}-1}{w_{\mathrm{pso}}} .
$$

Thus, the points corresponding to the PSO points located in the neighborhood of $(\omega, \bar{\phi})=(0,1)$ (where the PSO first order spectral radius is zero) lie in $(\omega, \bar{\phi}) \rightarrow$ $(-\infty,+\infty) \cup(\omega, \bar{\phi}) \rightarrow(+\infty,-\infty)$. This property means that the region of very fast trajectories' attenuation for the PSO and CC-PSO cases is not present for the CP-PSO algorithm, and thus, the CP-PSO version is expected to show greater exploratory capabilities. 
4. The same correspondence applies to the second order trajectories and the second order spectral radius.

These correspondences (31) make the algorithms linearly isomorphic, but when applied to real optimization problems they show different performances (as we will show in the section devoted to their numerical comparison) for two main reasons:

1. The introduction of a delay parameter increases the exploration and makes the algorithms different in the way they update the force terms. This is the case of CP-PSO with delay one.

2. The different way these algorithms update the positions and velocities of the particles.

\section{Numerical experiments using benchmark functions}

It is impossible to check exhaustively the performance of these new algorithms compared to GPSO for different benchmark functions, in different dimensions, varying all the interesting parameters concerning the first and second order trajectories in order to come up with some general recommendations about each algorithm. For this reason, the following strategy has been adopted:

1. We show that these algorithms, despite being linearly isomorphic, behave differently on several well-known benchmark functions. We perform this analysis for four different $(\omega, \bar{\phi})$ points belonging to the different zones of the first order stability region (Fernández Martínez et al. 2008), and their corresponding points which have been determined using (31). This analysis is done for several benchmark functions: DeJong, Ackley, Rastrigin, Rosenbrock, and Griewank. For the CP-PSO algorithm we also compare the zero and unit delay versions. ${ }^{2}$

2. We do numerical simulations for two well-known benchmark functions in two dimensions. We show that the performing points for all the family members are close to the intersection of the border of the second order stability and the median lines of the first order stability regions, where no temporal correlation between trajectories exists. These points can be analytically determined, and in the PSO case we show numerically that they perform as well as some popular parameter sets found in the literature. Finally, we analyze how these results are affected by an increase in the number of parameters of the cost function.

For comparison purposes, we have used the program PSOt published by Birge (2003) where we had implemented the CC-PSO and CP-PSO versions.

\subsection{Benchmark in corresponding points}

The aim of this section is to prove numerically that these PSO members have different performance when applied to benchmark functions. We have used four sets of $(w, \bar{\phi})$ points selected on the different regions of the first stability region:

- Complex zone, $(\omega, \bar{\phi})=(0.6,1.7)$

- Real symmetric zigzagging zone, $(\omega, \bar{\phi})=(0.5,2.95)$

\footnotetext{
${ }^{2}$ The zero delay CP version is linearly isomorphic to GPSO and CC-GPSO that have been deduced with zero delay.
} 
- Real asymmetric zigzagging zone, $(\omega, \bar{\phi})=(-0.5,0.5)$

- Real exponential decreasing zone, $(\omega, \bar{\phi})=(0.5,0.05)$

To find the corresponding $(\omega, \bar{\phi})$ points for the other family members, we have adopted the correspondences stated in (31).

Figure 7 shows the results obtained with different benchmark functions in ten dimensions (DeJong, Ackley, Rastrigin, Rosenbrock, and Griewank) adopting the point located on the
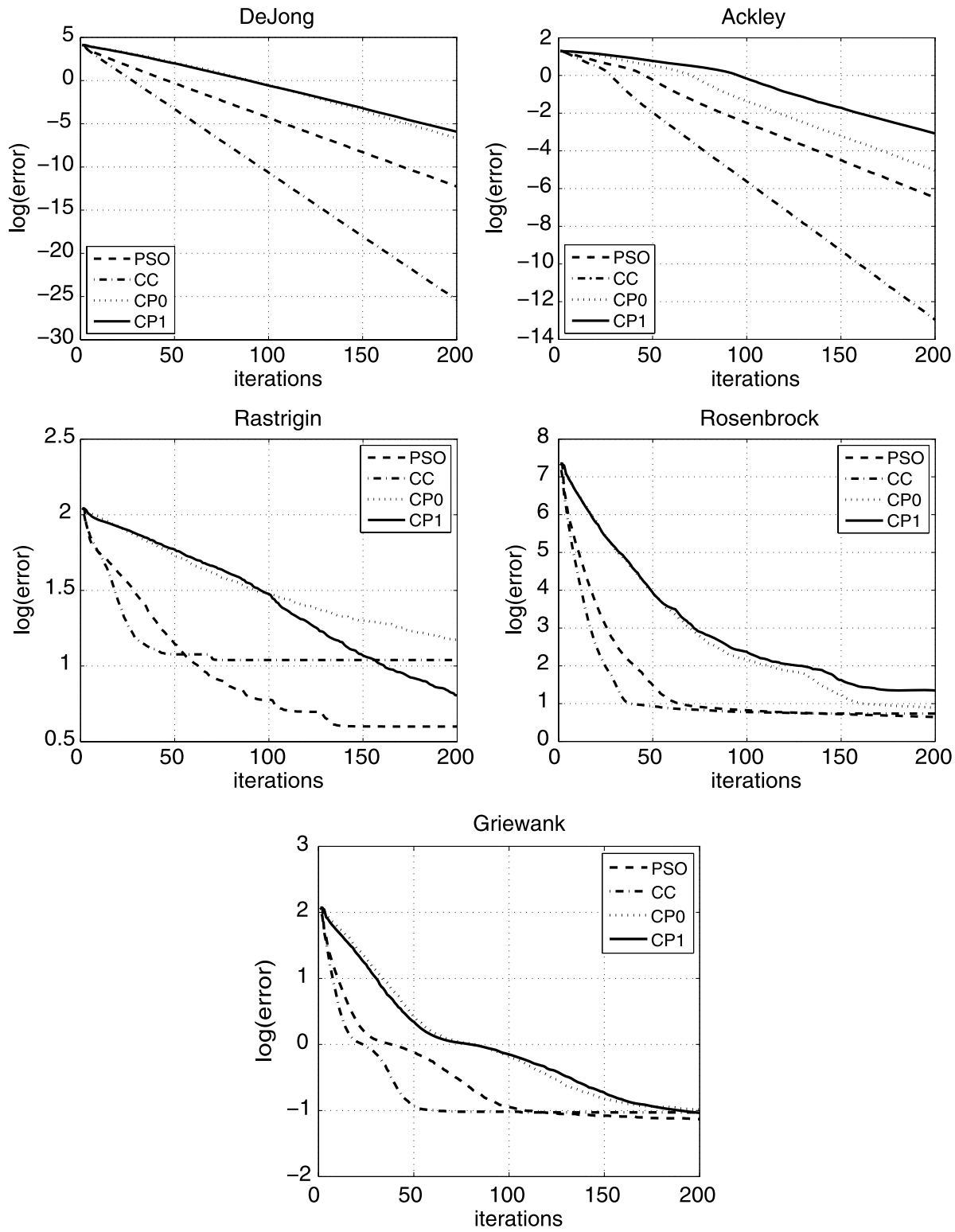

Fig. 7 Comparison (in $\log _{10}$ scale) of different algorithms in the corresponding points of Trelea point for different benchmark functions: DeJong, Ackley, Rastrigin, Rosenbrock, and Griewank 

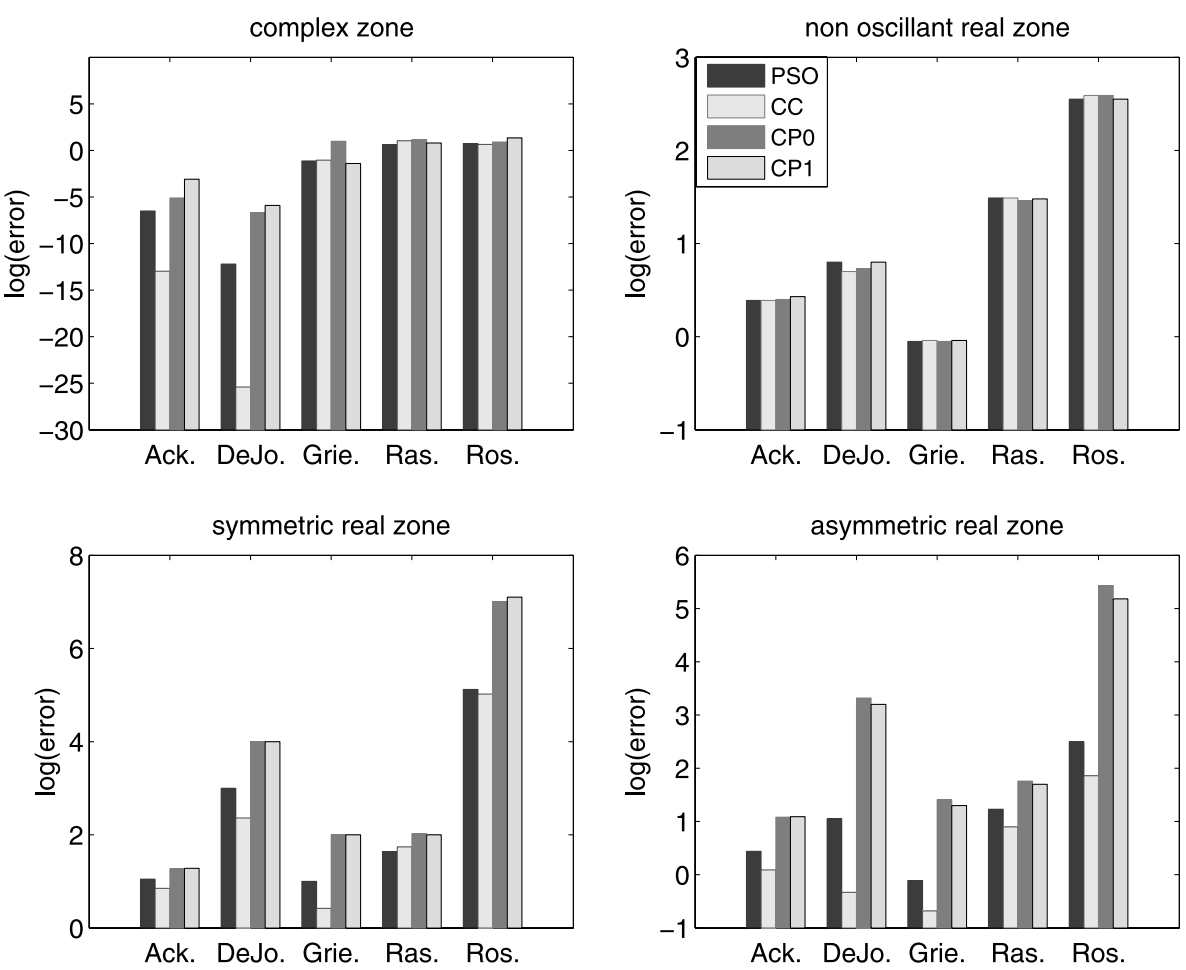

Fig. 8 Bar diagram showing the comparison of the minimum errors (in $\log _{10}$ scale) for corresponding points located in the four different zones of the first stability region. Note that the origin of the error bars is sometimes shifted to account for the negative values

complex zone proposed by Trelea (2003). The convergence curves shown are medians of 500 independent simulations, 100 particles, and 200 iterations. Figure 8 shows the error bar diagrams obtained for these benchmark functions with four different algorithms: PSO, CC-PSO, CP-PSO0, and CP-PSO1 (or simply CP-PSO).

Several conclusions can be obtained:

1. Results are very different for all the selected benchmark functions when the point $(w, \bar{\phi})$ is selected on the zones of high variability (complex zone or real zigzagging zones). In regions of low variability, the results are almost identical, that is, the algorithms are equivalent.

2. CC-PSO is the fastest algorithm in searching the global minimum area, ${ }^{3}$ and also shows almost systematically the lowest value of the cost function, except for the Rastrigin function. In this last case, PSO is the best performing algorithm.

3. The CP-PSO versions with delay zero (CP-PSO0) and the one with delay one (CP-PSO1) give similar results, but the exploration of the CP-PSO1 version is slightly greater because the center of attraction is delayed with respect to the trajectories. For all the benchmark functions, both CP-PSO versions show higher values of the interquartile curve as a function of the iterations. This measure of error dispersion (repeatability) can also be

\footnotetext{
${ }^{3}$ In this paper, we refer to minimization type problems.
} 
interpreted as a higher exploration capability of this algorithm. Thus, it can be concluded that the different behavior observed for the CP-PSO case is mainly due to the velocity discretization adopted. As mentioned before, this could be understood in terms of the Heisenberg uncertainty principle, since in the CP-PSO version positions and velocities are updated at the same time, and both cannot be known to arbitrary precision.

\subsection{Promising parameter sets}

The different PSO versions have been tested for different benchmark functions having both types of numerical difficulties commonly found in inverse problems: Rosenbrock (global minimum located in a flat narrow valley) and Griewank (many local minima) functions defined in dimension 2. In this section, we prove numerically that the results previously shown for the PSO version in Fernández Martínez and García Gonzalo (2008) do generalize to CC-PSO and CP-PSO.

Figure 9 shows for each algorithm the contour plots of the percentage of times when these algorithms arrive very close to the global minimum after a certain number of iterations (100). This numerical analysis is done for a lattice of $(\omega, \bar{\phi})$ point values located in the corresponding first order stability regions, over 50 different simulations. It can be observed that, in the three PSO versions, good parameter sets are close to the intersection of the border of the second order stability and the median lines of the first order stability regions, which are the lines where no temporal correlation exist between trajectories. For the PSO algorithm, it is important to note that there exists a region of parameter sets with negative inertia weight that also yields good results. The corresponding regions for CC-PSO and CP-PSO are located on the regions with $\omega<-1$ and $\omega>3$, respectively.

This analysis shows that all the PSO versions perform fairly well in a very broad area of the parameter space (inertia weight and mean acceleration).

The intersection between the line of temporal uncorrelation and the limit of second order stability ${ }^{4}$ gives the following promising parameter sets as a function of the time step, $\Delta t$, and $\alpha=\frac{a_{g}}{\bar{\phi}}$ :

1. $\operatorname{GPSO},(\omega, \bar{\phi})=\left(1-\frac{2\left(2-2 \alpha+\alpha^{2}\right)}{\left(8-2 \alpha+\alpha^{2}\right) \Delta t}, \frac{12}{\left(8-2 \alpha+\alpha^{2}\right) \Delta t^{2}}\right)$.

2. CC-PSO, $(\omega, \bar{\phi})=\left(1-\frac{2-2 \alpha+\alpha^{2}}{3 \Delta t}, \frac{2}{\Delta t^{2}}\right)$.

3. CP-PSO, $(w, \bar{\phi})=\left(1-\frac{2\left(2-2 \alpha+\alpha^{2}\right)}{\left(2 \alpha+4-\alpha^{2}\right) \Delta t}, \frac{12}{\left(2 \alpha+4-\alpha^{2}\right) \Delta t^{2}}\right)$.

4. $\beta$-PSO, $\left\{\begin{array}{l}\omega=1-\frac{2\left(2-2 \alpha+\alpha^{2}\right)}{\left(8-2 \alpha+\alpha^{2}-2\left(2-2 \alpha+\alpha^{2}\right) \beta\right) \Delta t}, \\ \bar{\phi}=\frac{12}{\left(8-2 \alpha+\alpha^{2}-2\left(2-2 \alpha+\alpha^{2}\right) \beta\right) \Delta t^{2}} .\end{array}\right.$

\footnotetext{
${ }^{4}$ The same can be done for any other second order spectral radius isoline.
} 

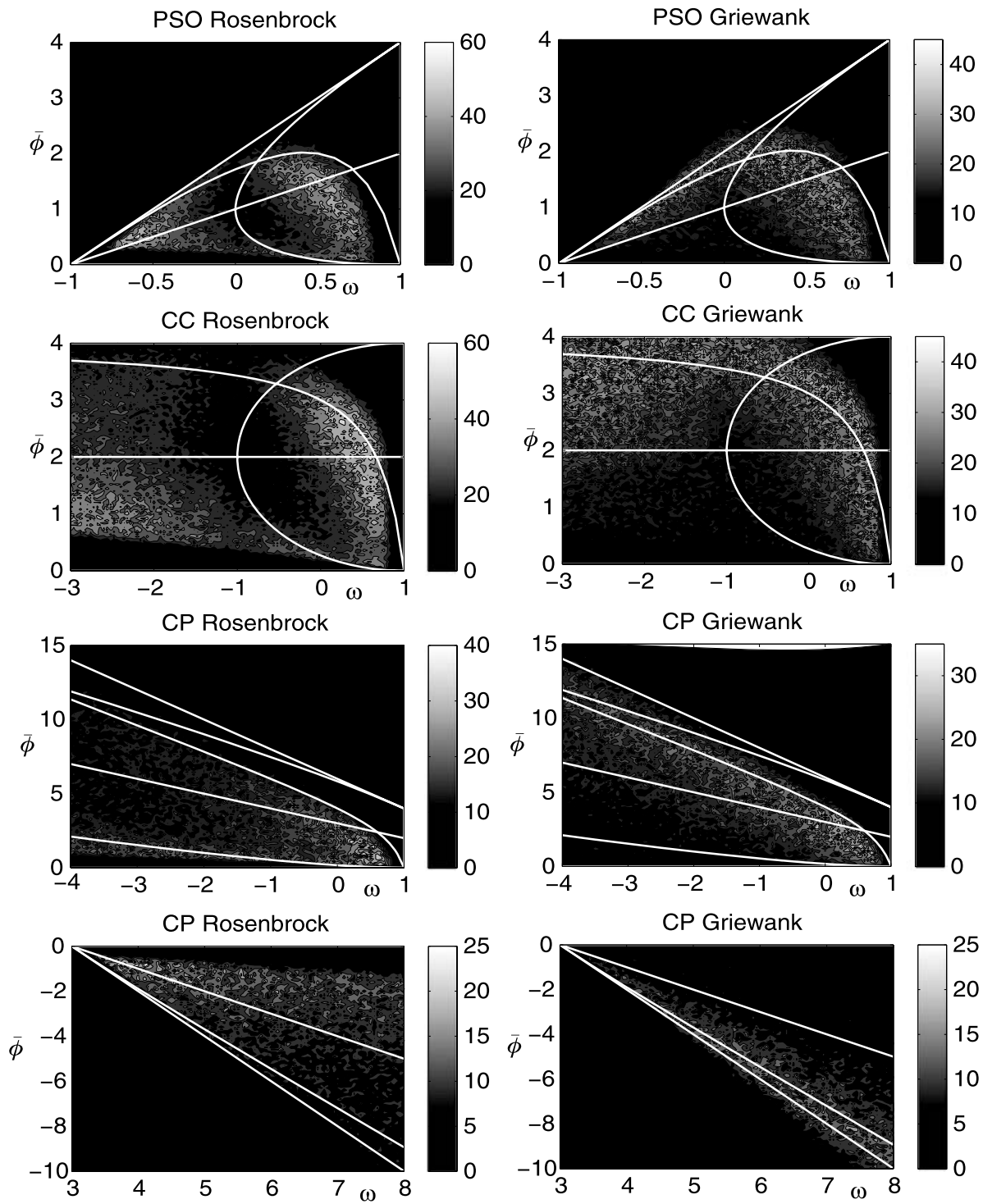

Fig. 9 Rosenbrock and Griewank functions in 2 dimensions: percentage of success for the different PSO versions. Observe that in the CP-PSO case these contour plots are made in the two disjoint zones of its first order stability region

Interesting points $(\omega, \bar{\phi})$ for different PSO versions can be deduced for any $\Delta t$ as a function of $\alpha$. Global and local accelerations can be calculated using the following formulas: $a_{g}=\alpha \bar{\phi}, a_{l}=\frac{2-\alpha}{\alpha} a_{g}$. In the PSO case, the intersection point I, $(\omega, \bar{\phi})=(0.714,1.714)$, can be compared with some popular parameter sets found in the literature (Carlisle and Dozier 2001; Clerc and Kennedy 2002; Trelea 2003) in terms of their performance, which is related to their respective first and second order trajectories and spectral radii. In what follows, it is 
important to understand that the second order spectral radius of matrix $A_{\sigma}, \rho_{\sigma}$, is a function of $(w, \bar{\phi}, \alpha, \Delta t)$, that is, $\left(w, a_{g}, a_{l}, \Delta t\right)$.

The following features are of interest:

1. The point $I$ is very close (in Euclidean distance) to the point proposed by Trelea (2003), $(\omega, \bar{\phi})=(0.6,1.7)$. The main difference between them is that Trelea point has a lower inertia weight and is located on the left, below the border of the second order stability region (10) on the spectral radius isoline, $\rho_{\sigma}=0.889$. Point I has a first order spectral radius of 0.845 . Trelea point has also a lower first order spectral radius, $\rho_{\mu}=0.775$.

2. The points proposed by Carlisle and Dozier (2001) and Clerc and Kennedy (2002) have the same first order spectral radius, $\rho_{\mu}=0.854$, corresponding to $(\omega, \bar{\phi})=$ $(0.729,1.494)$, but are located on different second order spectral radius isolines: 0.975 and 0.943, respectively. This implies that Carlisle and Dozier's point has a higher exploratory character than Clerc and Kennedy's point. These two points have a lower $\bar{\phi}$ than the point I, which is located on the border of the second order stability (spectral radius equal to 1 ).
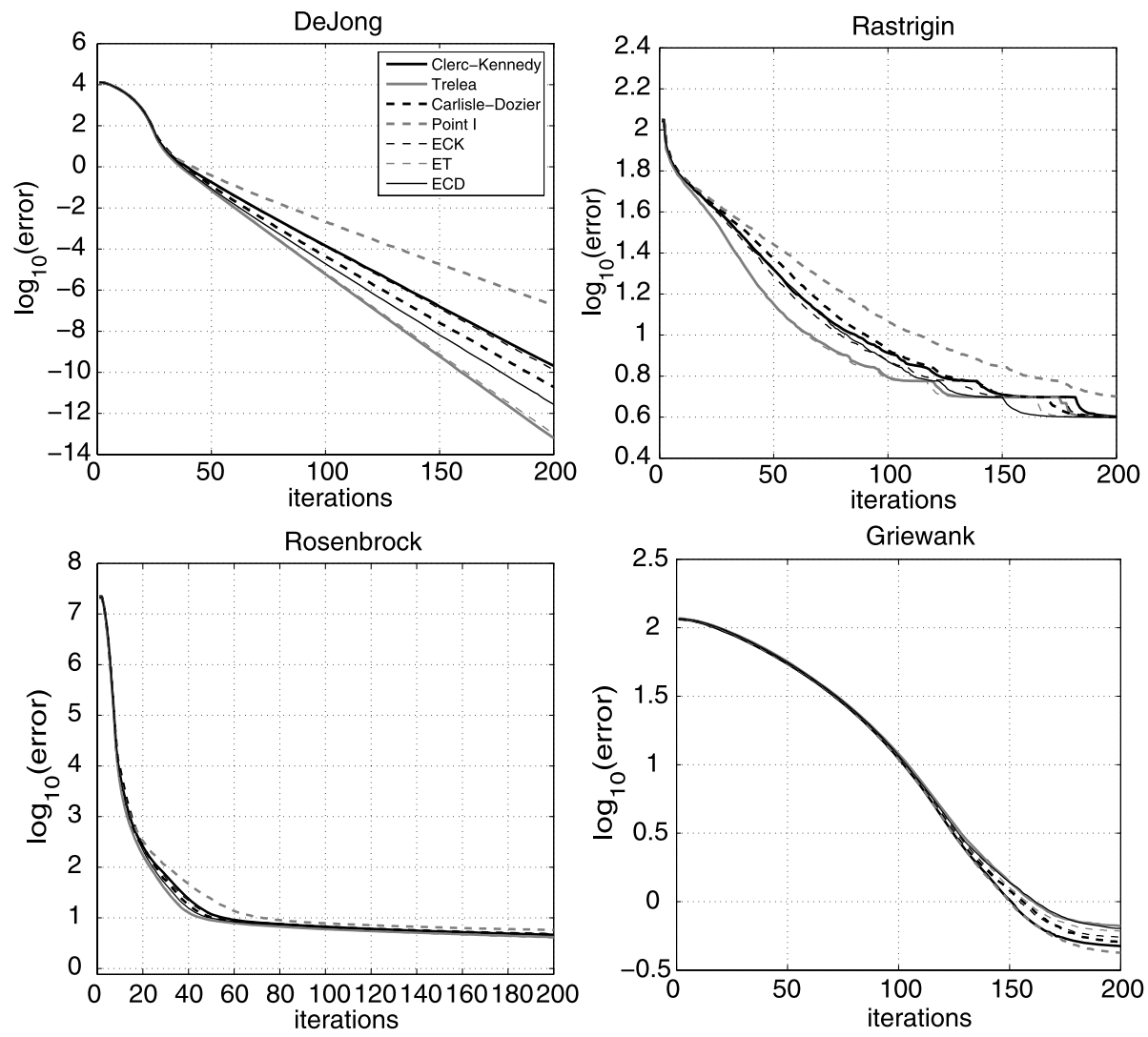

Fig. 10 Benchmark in 10 dimensions. Median convergence curves (in $\log _{10}$ scale) for different PSO parameter sets: DeJong, Rastrigin, Rosenbrock, and Griewank 

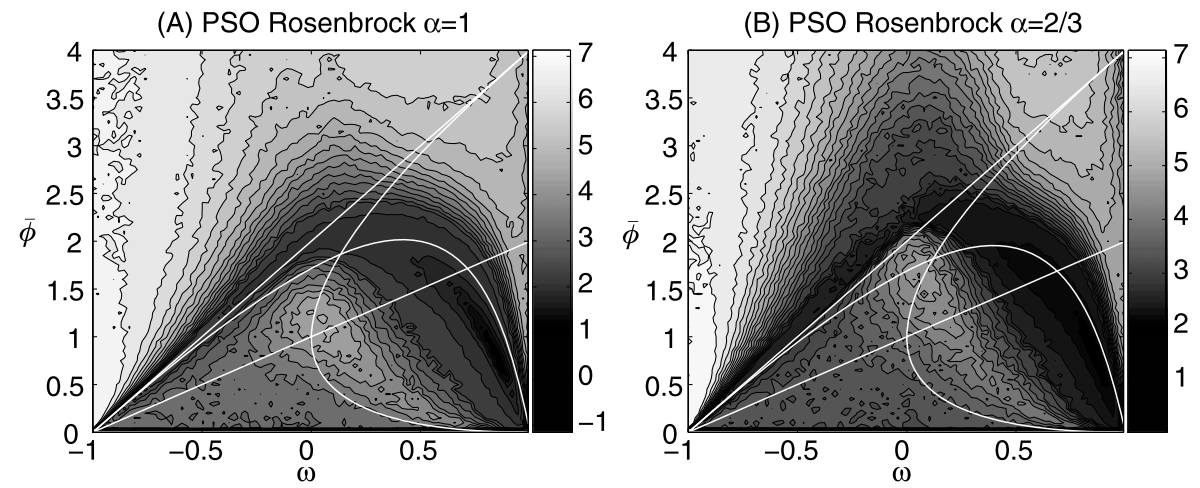

(C) PSO Rosenbrock $\alpha=4 / 3$

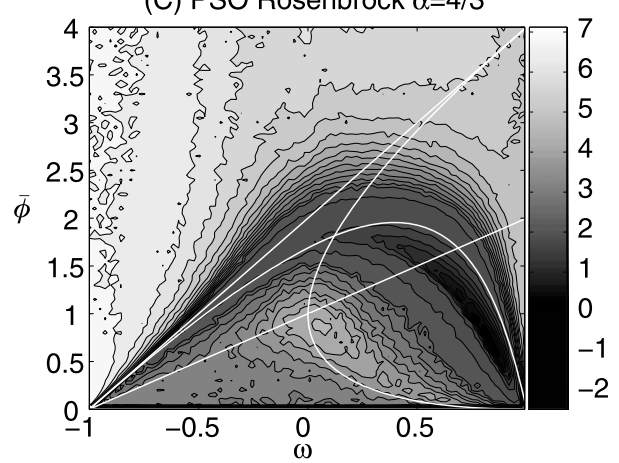

Fig. 11 Benchmark in 10 dimensions. PSO. Median error contour plot (in $\log _{10}$ scale) for the Rosenbrock function as a function of $\alpha:(\mathbf{A}) \alpha=1,(\mathbf{B}) \alpha=\frac{2}{3},(\mathbf{C}) \alpha=\frac{4}{3}$

Following the idea of being placed under the second order stability hyperbola, it is possible to propose other interesting points lying on the line of temporal uncorrelation between trajectories, $\bar{\phi}=1+\omega$, and having the same second order spectral radius as Trelea's, Clerc and Kennedy's, and Carlisle and Dozier's points $(0.889,0.975$ and 0.943$)$. These points are:

1. Equivalent Trelea point (ET), $\left(\omega, a_{g}, a_{l}\right)=(0.630,1.630,1630)$

2. Equivalent Clerc-Kennedy point (ECK), $\left(\omega, a_{g}, a_{l}\right)=(0.671,1.671,1.671)$

3. Equivalent Carlisle-Dozier point (ECD), $\left(\omega, a_{g}, a_{l}\right)=(0.664,1.055,2.272)$

Figure 10 shows, in the PSO case, the median convergence curves (after 500 simulations) for this set of promising points using the DeJong, Rastrigin, Rosenbrock and Griewank benchmark functions defined in ten dimensions, with 100 particles and 200 iterations. As expected, all the points perform equally well, confirming that there are no "magic PSO points" but only promising parameters sets. Also, when the numerical difficulties associated to the cost function decrease (DeJong case) the points with smaller second order spectral radius (less explorative), which are Trelea and ET points, seem to give the lower value of the cost function. Conversely, when the degree of numerical difficulties increases, points with higher second order spectral radius (such as point I) seem to give better results. This feature means that the exploration capabilities of the algorithm, which depend on the selected PSO parameters, must match the numerical difficulties of the optimization problem 

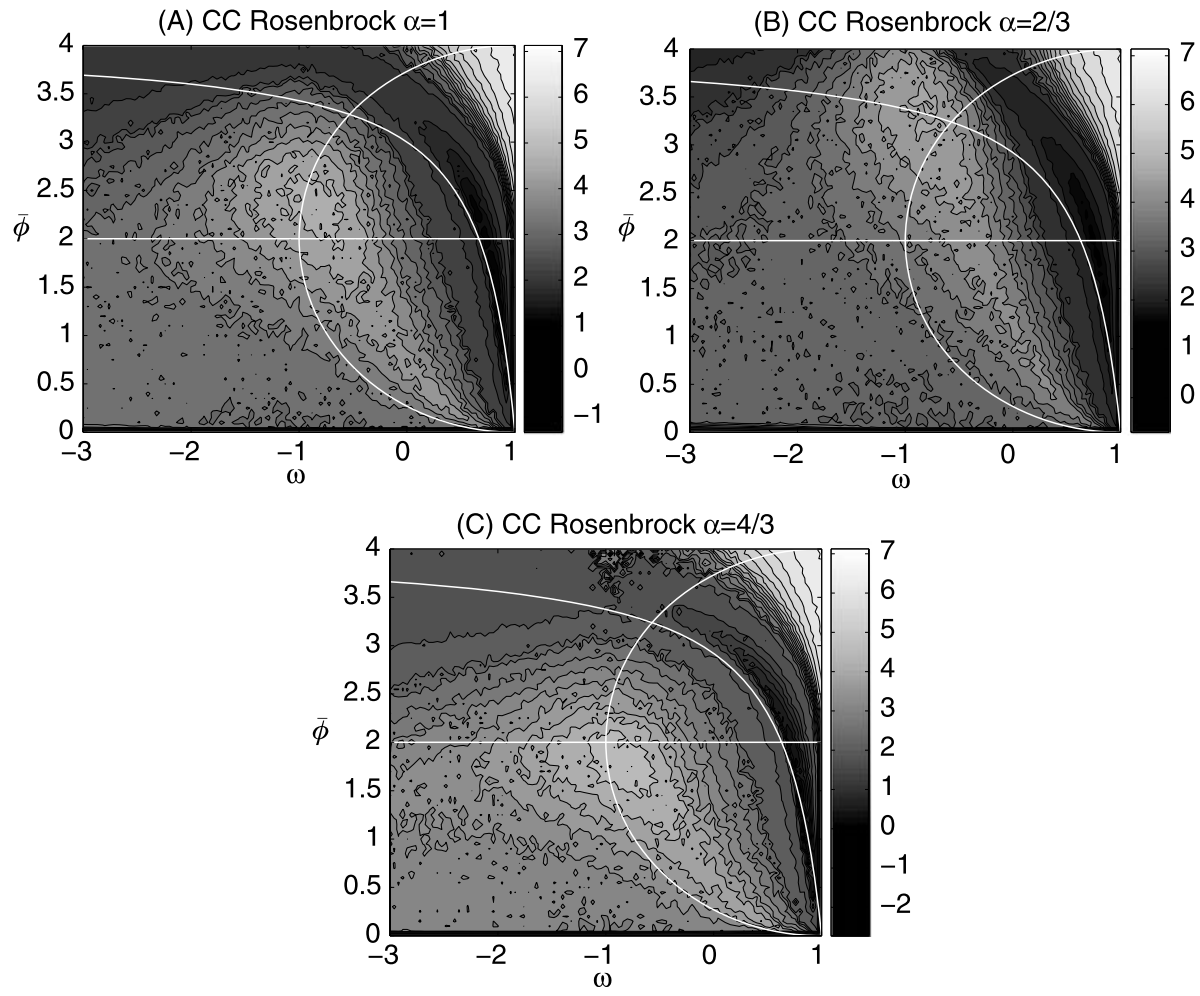

Fig. 12 Benchmark in 10 dimensions. CC-PSO. Median error contour plot (in $\log _{10}$ scale) for the Rosenbrock function as a function of $\alpha:(\mathbf{A}) \alpha=1,(\mathbf{B}) \alpha=\frac{2}{3},(\mathbf{C}) \alpha=\frac{4}{3}$

we need to solve, that is, the multimodality of the cost function and the dimensionality issue.

Finally, we have analyzed how these results change when the number of parameters is increased to ten. Generally speaking, when the number of parameters increases, the regions of good parameters move towards the inertia weight limit of stability, $w=1$, and lower total mean accelerations, where the temporal covariance between trajectories is positive. This feature is more clearly observed in the PSO case than in the other versions. All these features imply that the PSO variability has to be moderate to high in order to locate efficiently the minimum in a high dimensional space.

Figures 11 to 14 illustrate this fact. They show the numerical experiments performed in dimension 10 with the Rosenbrock and Griewank functions, for all the family members, and different values of $\alpha\left(1, \frac{2}{3}, \frac{4}{3}\right)$. To produce these median error plots we have performed 50 simulations with a swarm of 100 particles and 500 iterations at each $(\omega, \bar{\phi})$ point of a grid that includes the first order stability regions of the PSO family members. These figures confirm the conclusions that we have achieved in dimension 2. It is also possible to observe, in the CP-PSO case, the abrupt transition just above the limit of second order stability, from the region of good parameters to the zone of high errors. 

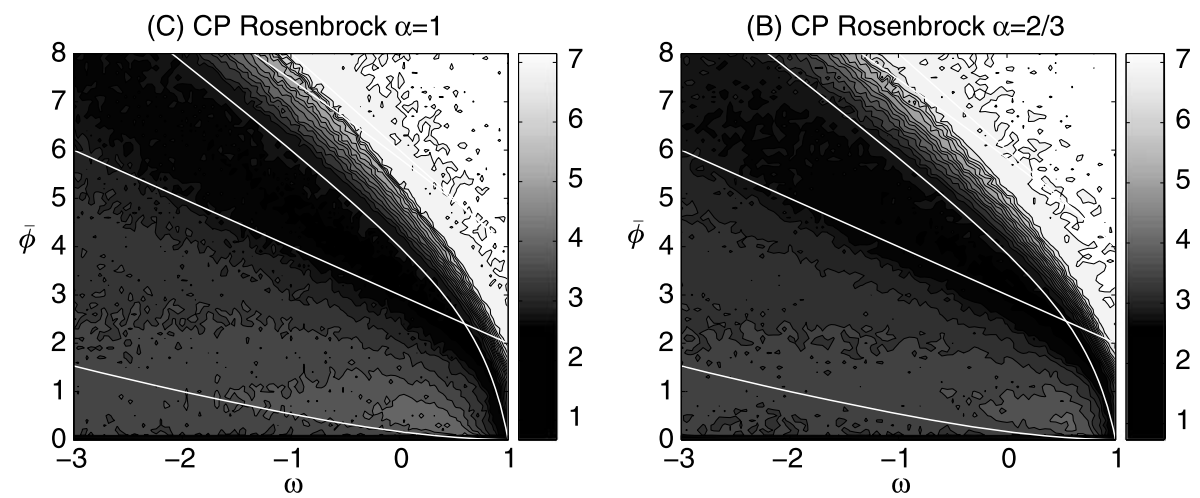

(C) CP Rosenbrock $\alpha=4 / 3$

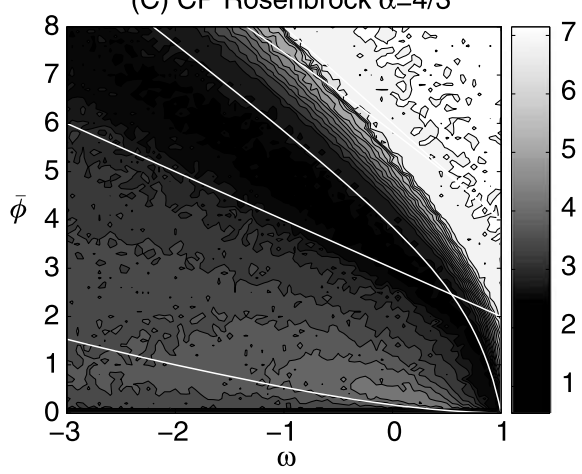

Fig. 13 Benchmark in 10 dimensions. CP-PSO. Median error contour plot (in $\log _{10}$ scale) for the Rosenbrock function as a function of $\alpha$ : (A) $\alpha=1,(\mathbf{B}) \alpha=\frac{2}{3},(\mathbf{C}) \alpha=\frac{4}{3}$

\section{Conclusions}

A family of PSO algorithms is presented and their first and second order stochastic stability is analyzed. These algorithms are linearly isomorphic when the same delay parameter is adopted. Nevertheless, they perform very differently when applied to different hard benchmark functions. Performance studies on synthetic functions also showed that good PSO parameter sets are close to the second order stability region, on the intersection of the corresponding median lines of the first order stability region, where no temporal correlation between trajectories exists. The main conclusion of this analysis is that there are no "magic" PSO tuning points deeply dependent on the kind of cost function, but regions where the different PSO versions have a higher probability to reach a good solution.

The behavior in terms of convergence rate and exploration capabilities of the PSO and CC-PSO versions is similar, but, in the benchmark functions we have analyzed, the CC-PSO and PSO versions fastest located the region of interesting models. Conversely, the CP-PSO version exhibited greater exploration capabilities. While solving real inverse problems, the exploration task of a global algorithm is very important to sample the space of possible solutions. Hybrid PSO versions could also help to optimally use the properties of each PSO member. 

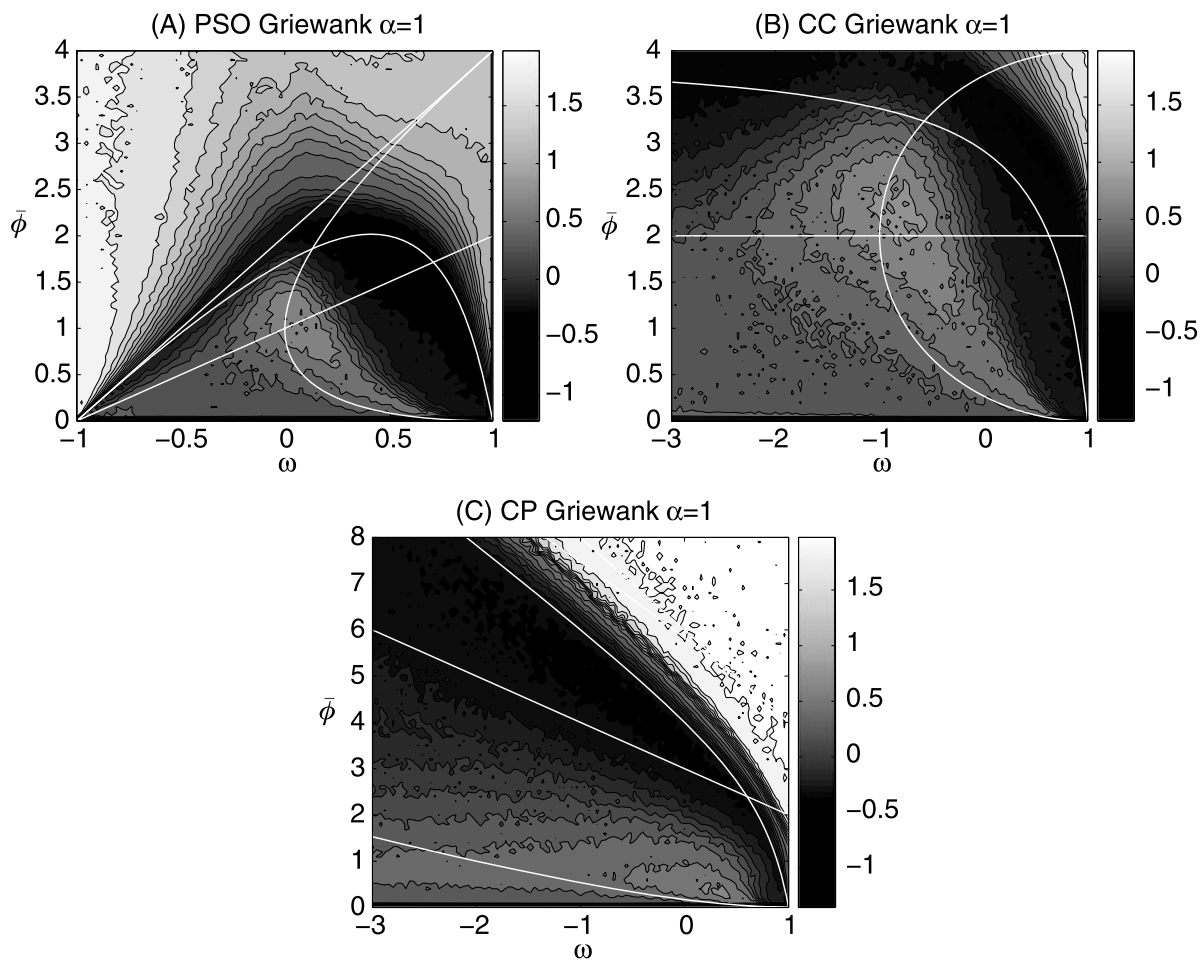

Fig. 14 Benchmark in 10 dimensions. Median error contour plot (in $\log _{10}$ scale) for the Griewank function and $\alpha=1$ : (A) PSO, (B) CC-PSO, (C) CP-PSO

Acknowledgements This work benefited from a sabbatical grant at University of California Berkeley (Department of Civil and Environmental Engineering) given by the "Secretaría de Estado de Universidades y de Investigación" of the Spanish Ministry of Science and Innovation. We would also like to acknowledge the professionalism and criticism of the peer reviews which helped us improve the quality of this manuscript, and particularly of Dr. Riccardo Poli for his interest in this research and for valuable criticism, suggestions and personal communications, for example, on the general relationships to determine corresponding points which are mentioned in this manuscript.

Open Access This article is distributed under the terms of the Creative Commons Attribution Noncommercial License which permits any noncommercial use, distribution, and reproduction in any medium, provided the original author(s) and source are credited.

\section{References}

Birge, B. (2003). PSOt, a particle swarm optimization toolbox for use with Matlab. IEEE swarm intelligence symposium, 2003. Proceedings of the 2003 IEEE, 24-26 April 2003, Indianapolis, USA (pp. 182-186). Digital Object Identifier doi:10.1109/SIS.2003.1202265.

Brandstätter, B., \& Baumgartner, U. (2002). Particle swarm optimization-mass-spring system analogon. IEEE Transaction on Magnetics, 38(2), 997-1000.

Carlisle, A., \& Dozier, G. (2001). An off-the-shelf PSO. In Proceedings of the workshop on particle swarm optimization, Purdue School of Engineering and Technology, Indianapolis (pp. 1-6).

Clerc, M. (2006). Stagnation analysis in particle swarm optimisation or what happens when nothing happens (Tech. Rep. CSM-460). Department of Computer Science, University of Essex, UK, August 2006. 
Clerc, M., \& Kennedy, J. (2002). The particle swarm—explosion, stability, and convergence in a multidimensional complex space. IEEE Transactions on Evolutionary Computation, 6(1), 58-73.

Fernández Martínez, J. L., \& García Gonzalo, E. (2008). The generalized PSO: a new door for PSO evolution. Journal of Artificial Evolution and Applications. doi:10.1155/2008/861275. Article ID 861275, 15 pages.

Fernández Martínez, J. L., \& García Gonzalo, E. (2009). Stochastic stability analysis of the linear continuous and discrete PSO models (Tech. Rep.). Department of Mathematics, University of Oviedo, Spain, June 2009.

Fernández Martínez, J. L., García Gonzalo, E., \& Fernández Alvarez, J. P. (2008). Theoretical analysis of particle swarm trajectories through a mechanical analogy. International Journal of Computational Intelligence Research, 4(2).

Jiang, M., Luo, Y. P., \& Yang, S. Y. (2007). Stochastic convergence analysis and parameter selection of the standard particle swarm optimization algorithm. Information Processing Letters, 2(1), 8-16.

Kadirkamanathan, V., Selvarajah, K., \& Fleming, P. J. (2006). Stability analysis of the particle dynamics in particle swarm optimizer. IEEE Transactions on Evolutionary Computation, 10(3), 245-255.

Poli, R. (2008). Dynamics and stability of the sampling distribution of particle swarm optimisers via moment analysis. Journal of Artificial Evolution and Applications. doi:10.1155/2008/761459. Article ID 761459, 10 pages.

Poli, R., \& Broomhead, D. (2007). Exact analysis of the sampling distribution for the canonical particle swarm optimiser and its convergence during stagnation. In Proceedings of the 9th genetic and evolutionary computation conference (GECCO'07) (pp. 134-141). New York: ACM.

Trelea, I. C. (2003). The particle swarm optimization algorithm: convergence analysis and parameter selection. Information Processing Letters, 85(6), 317-325.

Zheng, Y. L., Ma, L. H., Zhang, L. Y., \& Qian, J. X. (2003). On the convergence analysis and parameter selection in particle swarm optimisation. In Proceedings of the 2 nd international conference on machine learning and cybernetics (ICMLC'03) (Vol. 3, pp. 1802-1807). China.

Van den Bergh, F., \& Engelbrecht, A. P. (2006). A study of particle swarm optimization particle trajectories. Information Sciences, 176(8), 937-971. 